\title{
Fractional Brownian Motion in Finance
}

\author{
Bernt $\varnothing_{\mathrm{ksendal}}{ }^{1), 2)}$
}

Revised June 24, 2004

1) Center of Mathematics for Applications (CMA) Department of Mathematics, University of Oslo P.O. Box 1053 Blindern, N-0316, Oslo, Norway and

2) Norwegian School of Economics and Business Administration, Helleveien 30, N-5045, Bergen, Norway

\begin{abstract}
We give a survey of the stochastic calculus of fractional Brownian motion, and we discuss its applications to financial markets where the prices are described as solutions of stochastic differential equations driven by such processes.
\end{abstract}

MSC (2000): 60G15, 60G18, 60H40, 91B28, 91B70.

\section{Introduction}

How can we model (as a function of time)

(i) the levels of a river?

(ii) the characters of solar activity?

(iii) the widths of consecutive annual rings of a tree?

(iv) the outdoor temperature at a given point?

(v) the values of the $\log$ returns $h_{n}$, defined by

$$
h_{n}=\log \frac{S\left(t_{n}\right)}{S\left(t_{n-1}\right)}
$$

where $S(t)$ is the observed price at time $t$ of a given stock?

And how can we model

(vi) the turbulence in an incompressible fluid flow?

(vii) the electricity price in a liberated electricity market? 
The answer in all these cases is: By using a fractional Brownian motion!

The examples (i)-(iii), (v) and (vi) are taken from [Sh], example (iv) is from [BSZ] and example (vii) is from $[\mathrm{Si}]$.

This amazing range of potential applications makes fractional Brownian motion an interesting object to study. It is defined as follows:

Definition 1.1 Let $H \in(0,1)$ be a constant. The (1-parameter) fractional Brownian motion $(f B m)$ with Hurst parameter $H$ is the Gaussian process $B_{H}(t)=B_{H}(t, \omega), t \in \mathbb{R}, \omega \in \Omega$, satisfying

$$
B_{H}(0)=E\left[B_{H}(t)\right]=0 \quad \text { for all } t \in \mathbb{R}
$$

and

$$
E\left[B_{H}(s) B_{H}(t)\right]=\frac{1}{2}\left\{|s|^{2 H}+|t|^{2 H}-|s-t|^{2 H}\right\} ; \quad s, t \in \mathbb{R} .
$$

Here $E$ denotes the expectation with respect to the probability law $P$ for $\left\{B_{H}(t)\right\}_{t \in \mathbb{R}}=$ $\left\{B_{H}(t, \omega) ; t \in \mathbb{R}, \omega \in \Omega\right\}$, where $(\Omega, \mathcal{F})$ is a measurable space.

If $H=\frac{1}{2}$ then $B_{H}(t)$ coincides with the classical Brownian motion, denoted by $B(t)$.

If $H>\frac{1}{2}$ then $B_{H}(t)$ is persistent, in the sense that

$$
\rho_{n}:=E\left[B_{H}(1) \cdot\left(B_{H}(n+1)-B_{H}(n)\right)\right]>0 \quad \text { for all } n=1,2, \ldots
$$

and

$$
\sum_{n=1}^{\infty} \rho_{n}=\infty
$$

If $H<\frac{1}{2}$ then $B_{H}(t)$ is anti-persistent, in the sense that

$$
\rho_{n}<0 \quad \text { for all } n=1,2, \ldots
$$

In this case

$$
\sum_{n=1}^{\infty}\left|\rho_{n}\right|<\infty \quad([\mathrm{Sh}], \text { p. 233) }
$$

In the examples (i)-(vii) above, one would use $f B m$ with $H>\frac{1}{2}$ in (i)-(v) and with $H<\frac{1}{2}$ in (vi) and (vii).

Another important property of $f B m$ is self-similarity: For any $H \in(0,1)$ and $\alpha>0$ the law of $\left\{B_{H}(\alpha t)\right\}_{t \in \mathbb{R}}$ is the same as the law of $\left\{\alpha^{H} B_{H}(t)\right\}_{t \in \mathbb{R}}$.

In order to be able to apply $f B m$ to study the situations above we need a stochastic calculus for $f B m$. However, if $H \neq \frac{1}{2}$ then $B_{H}(t)$ is not a semimartingale, so one cannot use the general theory of stochastic calculus for semimartingales on $B_{H}(t)$. For example, it is not a priori clear what a stochastic integral of the form

$$
\int_{0}^{T} \phi(t, \omega) d B_{H}(t)
$$

should mean. The two most common constructions of such a stochastic integral are the following: 


\section{(I) The pathwise or forward integral}

This integral is denoted by

$$
\int_{0}^{T} \phi(t, \omega) d^{-} B_{H}(t)
$$

If the integrand $\phi(t, \omega)$ is caglad (left-continuous with right sided limits) then this integral can be defined by Riemann sums, as follows:

Let $0=t_{0}<t_{1}<\cdots<t_{N}=T$ be a partition of $[0, T]$. Put $\Delta t_{k}=t_{k+1}-t_{k}$ and define

$$
\int_{0}^{T} \phi(t, \omega) d^{-} B_{H}(t)=\lim _{\Delta t_{k} \rightarrow 0} \sum_{k=0}^{N-1} \phi\left(t_{k}\right) \cdot\left(B\left(t_{k+1}\right)-B\left(t_{k}\right)\right),
$$

if the limit exists (e.g. in probability). See Theorem 2.14.

Note that with this definition the integration takes place with respect to $t$ for each fixed "path" $\omega \in \Omega$. Therefore this integral is often called the pathwise integral. Using a classical integration theory due to Young one can prove that the pathwise integral (1.6) exists if the $p$-variation of $t \rightarrow \phi(t, \omega)$ is finite for all $p>(1-H)^{-1}$. See $[\mathrm{N}]$ and the references therein. Since $t \rightarrow B_{H}(t)$ has finite $q$-variation iff $q \geq \frac{1}{H}$, we see that if $H<\frac{1}{2}$ then this theory does not even include integrals like

$$
\int_{0}^{T} B_{H}(t) d^{-} B_{H}(t)
$$

For this reason one often assumes that $H>\frac{1}{2}$ when dealing with forward integrals with respect to $B_{H}(t)$. In general

$$
E\left[\int_{0}^{T} \phi(t, \omega) d^{-} B_{H}(t)\right] \neq 0,
$$

even if the forward integral belongs to $L^{1}(P)$.

For $H>\frac{1}{2}$ the forward integral obeys Stratonovich type of integration rules. For example, if $f \in C^{1}(\mathbb{R})$ and

$$
X_{t}:=\int_{0}^{t} \phi(s, \omega) d^{-} B_{H}(s) \quad \text { exists for all } t>0
$$

then

$$
f\left(X_{t}\right)=f(0)+\int_{0}^{t} f^{\prime}\left(X_{s}\right) d^{-} X_{s}
$$

where

$$
d^{-} X_{s}=\phi(s, \omega) d^{-} B_{H}(s) .
$$


(See e.g. [N] and also Theorem 3.16.) For this reason the forward integral is also sometimes called the Stratonovich integral with respect to $f B m$. As a special case of (1.8) we note that

$$
\int_{0}^{T} B_{H}(t) d^{-} B_{H}(t)=\frac{1}{2} B_{H}^{2}(T) \quad \text { for } H>\frac{1}{2} \text {. }
$$

Moreover, a slight extension of (1.8) gives that the unique solution $X_{t}$ of the fractional forward stochastic differential equation

$$
d^{-} X(t)=\alpha(t, \omega) X(t) d t+\beta(t, \omega) X(t) d^{-} B_{H}(t) ; \quad X(0)=x>0
$$

is

$$
X(t)=x \exp \left(\int_{0}^{t} \alpha(s, \omega) d s+\int_{0}^{t} \beta(s, \omega) d^{-} B_{H}(s)\right) \quad \text { for } H>\frac{1}{2},
$$

provided that the integrals on the right hand side exist.

\section{(II) The Skorohod (Wick-Itô) integral}

This integral is denoted by

$$
\int_{0}^{T} \phi(t, \omega) \delta B_{H}(t)
$$

It may be defined in terms of Riemann sums, as follows:

$$
\int_{0}^{T} \phi(t, \omega) \delta B_{H}(t)=\lim _{\Delta t_{k} \rightarrow 0} \sum_{k=0}^{N-1} \phi\left(t_{k}\right) \diamond\left(B\left(t_{k+1}\right)-B\left(t_{k}\right)\right),
$$

where $\diamond$ denotes the Wick product (see Theorem 2.11). Thus the difference between this integral and the forward integral is the use of the Wick product instead of the ordinary product in the Riemann sums (1.12) and (1.6), respectively.

The Skorohod integral behaves in many ways like the Itô integral of classical Brownian motion. For example, we have

$$
E\left[\int_{0}^{T} \phi(t, \omega) \delta B_{H}(t)\right]=0
$$

if the integral belongs to $L^{2}(P)$. Moreover, if $f \in C^{2}(\mathbb{R})$ then we have the following Itô type formula

$$
f\left(B_{H}(t)\right)=f(0)+\int_{0}^{t} f^{\prime}\left(B_{H}(s)\right) \delta B_{H}(s)+H \int_{0}^{t} f^{\prime \prime}\left(B_{H}(s)\right) s^{2 H-1} d s,
$$


valid for all $H \in(0,1)$, provided that the left hand side and the last term on the right hand side both belong to $L^{2}(P)$ (see [BØSW]). (See also $[\mathrm{B}]$, [vdH], $[\mathrm{H}]$ and $[\mathrm{M}]$ for related results. In $[\mathrm{DHP}]$ and $[\mathrm{B} \varnothing]$ Itô formulae for more general processes are proved, but valid only for $H>\frac{1}{2}$.)

Note that as a special case of (1.14) we get

$$
\int_{0}^{T} B_{H}(t) \delta B_{H}(t)=\frac{1}{2} B_{H}^{2}(T)-\frac{1}{2} T^{2 H}, \quad H \in(0,1) .
$$

The Wick-Skorohod-Itô analogue of (1.10) is the equation

$$
\delta X(t)=\alpha(t, \omega) X(t) d t+\beta(t, \omega) X(t) \delta B_{H}(t) ; \quad X(0)=x>0 .
$$

Assume that $\alpha(t, \omega)=\alpha$ and $\beta(t, \omega)=\beta$ are constants. Then by a slight extension of the Itô formula (1.14) one obtains that the unique solution of (1.16) is

$$
X(t)=x \exp \left(\beta B_{H}(t)+\alpha t-\frac{1}{2} \beta^{2} t^{2 H}\right) ; \quad H \in(0,1) .
$$

Note that if $H=\frac{1}{2}$ then the formulas (1.15) and (1.17) reduce to the formulas obtained by the Itô formula for the classical Brownian motion.

Later in this paper we will give a more detailed discussion about these two types of integration and their use in finance (Section 4).

But first we recall the mathematical foundation of fractional Brownian motion calculus based on white noise theory (Sections 2 and 3).

\section{Classical white noise theory and Hida-Malliavin calculus}

In this section we give a brief review of some fundamental concepts and results from classical white noise theory. We refer to [HØUZ], [HKPS] and [K] for more information.

Definition 2.1 Let $\mathcal{S}(\mathbb{R})$ be the Schwartz space of rapidly decreasing smooth functions on $\mathbb{R}$ and let $\Omega:=\mathcal{S}^{\prime}(\mathbb{R})$ be its dual, often called the space of tempered distributions. Then by the Bochner-Minlos theorem there exists a unique probability measure $P$ on the Borel subsets of $\Omega$ such that

$$
\int_{\Omega} e^{i\langle\omega, f\rangle} d P(\omega)=e^{-\frac{1}{2}\|f\|_{L^{2}(\mathbb{R})}^{2}} ; \quad f \in \mathcal{S}(\mathbb{R})
$$

where $i=\sqrt{-1},\|f\|_{L^{2}(\mathbb{R})}^{2}=\int_{\mathbb{R}} f(x)^{2} d x$ and $\langle\omega, f\rangle=\omega(f)$ denotes the action of $\omega \in \Omega=$ $\mathcal{S}^{\prime}(\mathbb{R})$ on $f \in \mathcal{S}(\mathbb{R})$. This measure $P$ is called the white noise probability measure.

From (2.1) it follows that

$$
E[\langle\omega, f\rangle]=0 \quad \text { for all } f \in \mathcal{S}(\mathbb{R}),
$$


where $E[\langle\omega, f\rangle]=E_{P}[\langle\omega, f\rangle]=\int_{\Omega}\langle\omega, f\rangle d P(\omega)$ denotes the expectation of $\langle\omega, f\rangle$ with respect to $P$. Moreover, (2.1) implies the isometry

$$
E\left[\langle\omega, f\rangle^{2}\right]=\|f\|_{L^{2}(\mathbb{R})}^{2} \quad \text { for all } f \in \mathcal{S}(\mathbb{R})
$$

Using (2.2) and (2.3) we can extend the definition of $\langle\omega, f\rangle$ from $\mathcal{S}(\mathbb{R})$ to $L^{2}(\mathbb{R})$ as follows:

If $f \in L^{2}(\mathbb{R})$ define

$$
\left.\langle\omega, f\rangle=\lim _{n \rightarrow \infty}\left\langle\omega, f_{n}\right\rangle \quad \text { (limit in } L^{2}(P)\right)
$$

where $f_{n} \in \mathcal{S}(\mathbb{R})$ and $f_{n} \rightarrow f$ in $L^{2}(\mathbb{R})$.

(It follows from (2.3) that the limit in (2.4) exists in $L^{2}(P)$ and is independent of the choice of the approximating sequence $\left\{f_{n}\right\}_{n=1}^{\infty} \subset \mathcal{S}(\mathbb{R})$.)

In particular, we can for each $t \in \mathbb{R}$ define

$$
\widetilde{B}(t):=\widetilde{B}(t, \omega):=\left\langle\omega, \mathcal{X}_{[0, t]}(\cdot)\right\rangle
$$

where

$$
\mathcal{X}_{[0, t]}(s)= \begin{cases}1 & \text { if } \quad 0 \leq s \leq t \\ -1 & \text { if } t \leq s \leq 0, \text { except } t=s=0 \\ 0 & \text { otherwise }\end{cases}
$$

By Kolmogorov's continuity theorem it can be proved that $\widetilde{B}(t)$ has a continuous version, which we will denote by $B(t)$. Then we see that $B(t)$ is a continuous Gaussian process with mean

$$
B(0)=E[B(t)]=0 \quad \text { for all } t
$$

and covariance

$$
E\left[B\left(t_{1}\right) B\left(t_{2}\right)\right]=\int_{\mathbb{R}} \mathcal{X}_{\left[0, t_{1}\right]}(s) \mathcal{X}_{\left[0, t_{2}\right]}(s) d s=\left\{\begin{array}{cl}
\min \left(\left[t_{1}|,| t_{2} \mid\right) ;\right. & \text { if } t_{1}, t_{2}>0 \\
0 & \text { otherwise }
\end{array}\right.
$$

Therefore $B(t)$ is a (classical) Brownian motion with respect to $P$.

Suppose $f(t)=\sum_{k} a_{k} \mathcal{X}_{\left[t_{k}, t_{k+1}\right)}(t)$ is a step function, where $t_{1}<t_{2}<\cdots<t_{N}$ and $a_{k} \in \mathbb{R}$.

Then by (2.5) and linearity we get

$$
\langle\omega, f\rangle=\sum_{k} a_{k}\left\langle\omega, \mathcal{X}_{\left[t_{k}, t_{k+1}\right)}(\cdot)\right\rangle=\sum_{k} a_{k}\left(B\left(t_{k+1}\right)-B\left(t_{k}\right)\right)=\int_{\mathbb{R}} f(t) d B(t) .
$$

By taking limits of such step functions we obtain that

$$
\langle\omega, f\rangle=\int_{\mathbb{R}} f(t) d B(t) \quad \text { for all } f \in L^{2}(\mathbb{R}) .
$$


In the following we let

$$
h_{n}(x):=(-1)^{n} e^{\frac{x^{2}}{2}} \frac{d^{n}}{d x^{n}}\left(e^{-\frac{x^{2}}{2}}\right) ; \quad n=0,1,2, \ldots
$$

be the Hermite polynomials and we let

$$
\xi_{n}(x):=\pi^{-\frac{1}{4}}((n-1) !)^{-\frac{1}{2}} h_{n-1}(\sqrt{2} x) e^{-\frac{x^{2}}{2}} ; \quad n=1,2, \ldots
$$

be the Hermite functions. Then $\left\{\xi_{n}\right\}_{n=1}^{\infty}$ consitutes an orthonormal basis for $L^{2}(\mathbb{R})$.

The first Hermite polynomials are: $h_{0}(x)=1, h_{1}(x)=x, h_{2}(x)=x^{2}-1, h_{3}(x)=$ $x^{3}-3 x, \ldots$

Let $\mathcal{J}$ be the set of all multi-indices $\alpha=\left(\alpha_{1}, \alpha_{2}, \ldots\right)$ of finite length (i.e. $\alpha_{k}=0$ for all $k$ large enough), with $\alpha_{i} \in \mathbb{N} \cup\{0\}=\{0,1,2, \ldots\}$ for all $i$. For $\alpha=\left(\alpha_{1}, \ldots, \alpha_{m}\right) \in \mathcal{J}$ define

$$
\mathcal{H}_{\alpha}(\omega)=h_{\alpha_{1}}\left(\left\langle\omega, \xi_{1}\right\rangle\right) h_{\alpha_{2}}\left(\left\langle\omega, \xi_{2}\right\rangle\right) \ldots h_{\alpha_{m}}\left(\left\langle\omega, \xi_{m}\right\rangle\right) .
$$

For example, if we put

$$
\varepsilon^{(k)}=(0,0, \ldots, 1) \in \mathbb{R}^{k} \quad \text { (the } k^{\prime} \text { th unit vector) }
$$

then we see that

$$
\mathcal{H}_{\varepsilon^{(k)}}(\omega)=h_{1}\left(\left\langle\omega, \xi_{k}\right\rangle\right)=\left\langle\omega, \xi_{k}\right\rangle=\int_{\mathbb{R}} \xi_{k}(t) d B(t) .
$$

It is a fundamental fact that the family $\left\{\mathcal{H}_{\alpha}\right\}_{\alpha \in \mathcal{J}}$ constitutes an orthogonal basis for $L^{2}(P)$. Indeed, we have:

Theorem 2.2 (The Wiener-Itô chaos expansion (I)) Let $F \in L^{2}(P)$. Then there exists a unique family $\left\{c_{\alpha}\right\}_{\alpha \in \mathcal{J}}$ of constants $c_{\alpha} \in \mathbb{R}$ such that

$$
F(\omega)=\sum_{\alpha \in \mathcal{J}} c_{\alpha} \mathcal{H}_{\alpha}(\omega) \quad \text { (convergence in } L^{2}(P) \text { ). }
$$

Moreover, we have the isometry

$$
E\left[F^{2}\right]=\sum_{\alpha \in \mathcal{J}} c_{\alpha}^{2} \alpha !
$$

where $\alpha !=\alpha_{1} ! \alpha_{2} ! \ldots \alpha_{m} !$ if $\alpha=\left(\alpha_{1}, \ldots, \alpha_{m}\right) \in \mathcal{J}$.

Example 2.3 For each $t \in \mathbb{R}$ the random variable $F(\omega)=B(t, \omega)$ belongs to $L^{2}(P)$. Its chaos expansion is

$$
\begin{aligned}
B(t) & =\left\langle\omega, \mathcal{X}_{[0, t]}(\cdot)\right\rangle=\left\langle\omega, \sum_{k=1}^{\infty}\left(\mathcal{X}_{[0, t]}, \xi_{k}\right)_{L^{2}(\mathbb{R})} \xi_{k}\right\rangle \\
& =\sum_{k=1}^{\infty}\left(\mathcal{X}_{[0, t]}, \xi_{k}\right)_{L^{2}(\mathbb{R})}\left\langle\omega, \xi_{k}\right\rangle=\sum_{k=1}^{\infty} \int_{0}^{t} \xi_{k}(s) d s \mathcal{H}_{\varepsilon^{(k)}}(\omega)
\end{aligned}
$$


where in general

$$
(f, g)_{L^{2}(\mathbb{R})}=\int_{\mathbb{R}} f(t) g(t) d t .
$$

We now use Theorem 2.2 to define stochastic test functions and stochastic distributions, as follows:

In the following we use the notation

$$
(2 \mathbb{N})^{\gamma}:=(2 \cdot 1)^{\gamma_{1}}(2 \cdot 2)^{\gamma_{2}} \ldots(2 \cdot m)^{\gamma_{m}}
$$

if $\gamma=\left(\gamma_{1}, \ldots, \gamma_{m}\right) \in \mathcal{J}$.

Definition 2.4 a) The space $(\mathcal{S})$ of Hida test functions is the set of all $\psi \in L^{2}(P)$ whose expansion

$$
\psi(\omega)=\sum_{\alpha \in \mathcal{J}} a_{\alpha} \mathcal{H}_{\alpha}(\omega)
$$

satisfies

$$
\sum_{\alpha \in \mathcal{J}} a_{\alpha}^{2} \alpha !(2 \mathbb{N})^{\alpha k}<\infty \quad \text { for all } k=1,2, \ldots
$$

b) The space $(\mathcal{S})^{*}$ of Hida distributions is the set of all formal expansions

$$
G(\omega)=\sum_{\alpha \in \mathcal{J}} b_{\alpha} \mathcal{H}_{\alpha}(\omega)
$$

such that

$$
\sum_{\alpha \in \mathcal{J}} b_{\alpha}^{2} \alpha !(2 \mathbb{N})^{-q \alpha}<\infty \quad \text { for some } q \in \mathbb{N}
$$

$W e$ equip $(\mathcal{S})$ with the projective topology and $(\mathcal{S})^{*}$ with the inductive topology. Then $(\mathcal{S})^{*}$ becomes the dual of $(\mathcal{S})$ and the action of $G \in(\mathcal{S})^{*}$ on $\psi \in(S)$ is given by

$$
\langle G, \psi\rangle=\langle G, \psi\rangle_{(\mathcal{S})^{*},(\mathcal{S})}=\sum_{\alpha \in \mathcal{J}} \alpha ! a_{\alpha} b_{\alpha} .
$$

Note that

$$
(\mathcal{S}) \subset L^{2}(P) \subset(\mathcal{S})^{*}
$$

Moreover, if $G \in L^{2}(P)$ then

$$
\langle G, \psi\rangle=E[G \cdot \psi] \quad \text { for all } \psi \in(\mathcal{S}) .
$$


Definition 2.5 (Integration in $\left.(\mathcal{S})^{*}\right) \quad$ Suppose $Z: \mathbb{R} \rightarrow(\mathcal{S})^{*}$ has the property that

$$
\langle Z(t), \psi\rangle \in L^{2}(\mathbb{R}, d t) \quad \text { for all } \psi \in(\mathcal{S}) .
$$

Then the integral

$$
\int_{\mathbb{R}} Z(t) d t
$$

is defined to be the unique element of $(\mathcal{S})^{*}$ such that

$$
\left\langle\int_{\mathbb{R}} Z(t) d t, \psi\right\rangle=\int_{\mathbb{R}}\langle Z(t), \psi\rangle d t \quad \text { for all } \psi \in(\mathcal{S}) .
$$

Such functions $Z(t)$ are called integrable in $(\mathcal{S})^{*}$.

Example 2.6 (White noise) Define

$$
W(t)=\sum_{k=1}^{\infty} \xi_{k}(t) \mathcal{H}_{\varepsilon(k)}(\omega) ; \quad t \in \mathbb{R} .
$$

Then by Definition 2.4b we see that $W(t) \in(\mathcal{S})^{*}$ for all $t$. Moreover

$$
\int_{0}^{t} W(s) d s=\sum_{k=1}^{\infty} \int_{0}^{t} \xi_{k}(s) d s \mathcal{H}_{\varepsilon^{(k)}}(\omega)=B(t),
$$

by Example 2.3. In other words, the function $t \rightarrow B(t)$ is differentiable in $(\mathcal{S})^{*}$ and

$$
\frac{d}{d t} B(t)=W(t) \quad \text { in }(\mathcal{S})^{*} .
$$

This justifies the name white noise for $W(t)$.

We now recall the definition of the Wick product, which was originally introduced by the physicist G. Wick in the early 1950's as a renormalization operation in quantum physics, but has later turned out to be central in stochastic analysis as well:

Definition 2.7 (The Wick product) Let

$$
F(\omega)=\sum_{\alpha \in \mathcal{J}} a_{\alpha} \mathcal{H}_{\alpha}(\omega) \in(\mathcal{S})^{*} \quad \text { and } \quad G(\omega)=\sum_{\beta \in \mathcal{J}} b_{\beta} \mathcal{H}_{\beta}(\omega) \in(\mathcal{S})^{*} .
$$

Then the Wick product of $F$ and $G, F \diamond G$, is defined by

$$
(F \diamond G)(\omega)=\sum_{\alpha, \beta \in \mathcal{J}} a_{\alpha} b_{\beta} \mathcal{H}_{\alpha+\beta}(\omega)=\sum_{\gamma \in \mathcal{J}}\left(\sum_{\alpha+\beta=\gamma} a_{\alpha} b_{\beta}\right) \mathcal{H}_{\gamma}(\omega) .
$$


One can easily verify that the Wick product is a commutative, associative and distributive (over addition) binary operation on both $(\mathcal{S})$ and on $(\mathcal{S})^{*}$. Moreover, note that

$$
F \diamond G=F \cdot G \quad \text { if either } F \text { or } G \text { is deterministic. }
$$

Example 2.8 If $F(\omega)=\int_{\mathbb{R}} f(t) d B(t)$ and $G(\omega)=\int_{\mathbb{R}} g(t) d B(t)$ with $f, g \in L^{2}(\mathbb{R})$ (deterministic), then

$$
F \diamond G=F \cdot G-(f, g)
$$

where

$$
(f, g)=(f, g)_{L^{2}(\mathbb{R})} .
$$

Proof. Using (2.28) and that $h_{2}(x)=x^{2}-1$ we get

$$
\begin{aligned}
F \diamond G & =\langle\omega, f\rangle \diamond\langle\omega, g\rangle \\
& =\left(\sum_{k=1}^{\infty}\left(f, \xi_{k}\right)\left\langle\omega, \xi_{k}\right\rangle\right) \diamond\left(\sum_{\ell=1}^{\infty}\left(g, \xi_{\ell}\right)\left\langle\omega, \xi_{\ell}\right\rangle\right) \\
& =\sum_{k, \ell=1}^{\infty}\left(f, \xi_{k}\right)\left(g, \xi_{\ell}\right) \mathcal{H}_{\varepsilon^{(k)}+\varepsilon^{(\ell)}} \\
& =\sum_{k \neq \ell}^{\infty}\left(f, \xi_{k}\right)\left(g, \xi_{\ell}\right) \mathcal{H}_{\varepsilon^{(k)}} \mathcal{H}_{\varepsilon^{(\ell)}}+\sum_{k=1}^{\infty}\left(f, \xi_{k}\right)\left(g, \xi_{k}\right) h_{2}\left(\left\langle\omega, \xi_{k}\right\rangle\right) \\
& =\sum_{k, \ell=1}^{\infty}\left(f, \xi_{k}\right)\left(g, \xi_{\ell}\right) \mathcal{H}_{\varepsilon^{(k)}} \mathcal{H}_{\varepsilon^{(\ell)}}-\sum_{k=1}^{\infty}\left(f, \xi_{k}\right)\left(g, \xi_{k}\right) \\
& =\langle\omega, f\rangle \cdot\langle\omega, g\rangle-(f, g) .
\end{aligned}
$$

One reason for the importance of the Wick product is the following result (we refer to [HØUZ] for a proof and more information):

Theorem 2.9 Suppose that $Y(t, \omega)$ is a stochastic process which is Skorohod integrable. Then $Y(t) \diamond W(t)$ is integrable in $(\mathcal{S})^{*}$ and

$$
\int_{\mathbb{R}} Y(t) \delta B(t)=\int_{\mathbb{R}} Y(t) \diamond W(t) d t,
$$

where the left hand side denotes the Skorohod integral of $Y(\cdot)$ with respect to $B(\cdot)$.

The Skorohod integral is an extension of the classical Itô integral, in the sense that if $Y(t, \omega)$ is measurable w.r.t. the $\sigma$-algebra $\mathcal{F}_{t}$ generated by $B(s, \omega) ; s \leq t$, for all $t$ (i.e. if $Y(\cdot)$ is $\mathcal{F}_{t^{-}}$adapted) and

$$
E\left[\int_{0}^{T} Y^{2}(t, \omega) d t\right]<\infty
$$


then

$$
\int_{0}^{T} Y(t) \delta B(t)=\int_{0}^{T} Y(t) d B(t), \quad \text { the classical Itô integral. }
$$

The integral on the right hand side of (2.31) may exist even if $Y$ is not Skorohod integrable. Therefore we may regard the right hand side of (2.31) as an extension of the Skorohod integral and we call it the extended Skorohod integral. We will use the same notation

$$
\int_{\mathbb{R}} Y(t) \delta B(t)
$$

for the extended Skorohod integral.

Example 2.10 Using Wick calculus in $(\mathcal{S})^{*}$ we get

$$
\begin{aligned}
\int_{0}^{T} B(T) \delta B(t) & =\int_{0}^{T} B(T) \diamond W(t) d t=B(T) \diamond \int_{0}^{T} W(t) d t \\
& =B(T) \diamond B(T)=B^{2}(T)-T,
\end{aligned}
$$

by Example 2.8 with $f=g=\mathcal{X}_{[0, T]}$.

The following result gives a useful interpretation of the Skorohod integral as a limit of Riemann sums:

Theorem 2.11 Let $Y:[0, T] \rightarrow(\mathcal{S})^{*}$ be a caglad function, i.e. $Y(t)$ is left-continuous with right sided limits. Then $Y$ is Skorohod integrable over $[0, T]$ and

$$
\int_{\mathbb{R}} Y(t) \delta B(t)=\lim _{\Delta t_{j} \rightarrow 0} \sum_{j=0}^{N-1} Y\left(t_{j}\right) \diamond\left(B\left(t_{j+1}\right)-B\left(t_{j}\right)\right)
$$

where the limit is taken in $(\mathcal{S})^{*}$ and $0=t_{0}<t_{1}<\cdots<t_{n}=T$ is a partition of $[0, T]$, $\Delta t_{j}=t_{j+1}-t_{j}, j=0, \ldots, N-1$.

Proof. This is an easy consequence of Theorem 2.9 .

We also note the following:

Theorem 2.12 Let $Y: \mathbb{R} \rightarrow(\mathcal{S})^{*}$. Suppose $Y(t)$ has the expansion

$$
Y(t)=\sum_{\alpha \in \mathcal{J}} c_{\alpha}(t) \mathcal{H}_{\alpha}(\omega) ; \quad t \in \mathbb{R}
$$

where

$$
c_{\alpha} \in L^{2}(\mathbb{R}) \quad \text { for all } \alpha \in \mathcal{J} \text {. }
$$


Then

$$
\int_{\mathbb{R}} Y(t) \delta B(t)=\sum_{\alpha \in \mathcal{J}} \sum_{k \in \mathbb{N}}\left(c_{\alpha}, \xi_{k}\right) \mathcal{H}_{\alpha+\varepsilon^{(k)}}(\omega),
$$

provided that the right hand side converges in $(\mathcal{S})^{*}$. In particular, if $\int_{\mathbb{R}} Y(t) \delta B(t) \in L^{2}(P)$ then

$$
E\left[\int_{\mathbb{R}} Y(t) \delta B(t)\right]=0
$$

\section{The forward integral}

We have already noted that the Skorohod integral is an extension of the classical Itô integral to integrands which are not necessarily adapted. There is another natural extension of this type, called the forward integral, which we now define:

Definition 2.13 The forward integral of a function $Y: \mathbb{R} \rightarrow(\mathcal{S})^{*}$ is defined by

$$
\int_{\mathbb{R}} Y(t) d^{-} B(t)=\lim _{\varepsilon \rightarrow 0} \int_{\mathbb{R}} Y(t) \frac{B(t+\varepsilon)-B(t)}{\varepsilon} d t
$$

provided that the limit exists in $(\mathcal{S})^{*}$.

We refer to $[\mathrm{NP}]$ and $[\mathrm{RV}]$ for more information about the forward integral. At this stage we will settle with the following result, which gives an easy comparison with the Skorohod integral (see Theorem 2.11).

Theorem 2.14 Suppose that $Y:[0, T] \rightarrow(\mathcal{S})^{*}$ is caglad and forward integrable over $[0, T]$. Then

$$
\int_{0}^{T} Y(t) d^{-} B(t)=\lim _{\Delta t_{j} \rightarrow 0} \sum_{j=0}^{N-1} Y\left(t_{j}\right) \cdot\left(B\left(t_{j+1}\right)-B\left(t_{j}\right)\right) \quad\left(\text { limit in }(\mathcal{S})^{*}\right) .
$$

Proof. This follows by a Fubini argument. See e.g. (2.24) in [B $\varnothing]$ for a proof.

We say that $X(t)$ is a forward Itô process if

$$
X(t)=x+\int_{0}^{t} u(s, \omega) d s+\int_{0}^{t} v(s, \omega) d^{-} B(s) ; \quad t \geq 0
$$

for some measurable processes $u(s, \omega), v(s, \omega) \in \mathbb{R}$ (not necessarily adapted) such that

$$
\int_{0}^{t}|u(s, \omega)| d s<\infty
$$


and the Itô forward integral

$$
\int_{0}^{t} v(s, \omega) d^{-} B(s)
$$

exists for all $t>0$. In that case we use the shorthand notation

$$
d^{-} X(t)=u(t) d t+v(t) d^{-} B(t) ; \quad X(0)=x
$$

for the integral equation (2.39).

For such processes we have the following Itô formula:

Theorem 2.15 [RV] (Itô formula for forward processes) Let $f \in C^{2}(\mathbb{R})$ and define

$$
Y(t)=f(X(t))
$$

Then $Y(t)$ is a forward Itô process and

$$
d^{-} Y(t)=f^{\prime}(X(t)) d^{-} X(t)+\frac{1}{2} f^{\prime \prime}(X(t)) v^{2}(t) d t
$$

\section{Stochastic differentiation}

We now make use of our explicit knowledge of the space $\Omega=\mathcal{S}^{\prime}(\mathbb{R})$ to define differentiation with respect to $\omega$, as follows:

Definition 2.16 a) Let $F: \Omega \rightarrow \mathbb{R}, \gamma \in L^{2}(\mathbb{R})$. Then the directional derivative of $F$ in the direction $\gamma$ is defined by

$$
D_{\gamma} F(\omega)=\lim _{\varepsilon \rightarrow 0} \frac{F(\omega+\varepsilon \gamma)-F(\omega)}{\varepsilon}
$$

provided that the limit exists in $(\mathcal{S})^{*}$.

b) Suppose there exists a function $\psi: \mathbb{R} \rightarrow(S)^{*}$ such that

$$
D_{\gamma} F(\omega)=\int_{\mathbb{R}} \psi(t) \gamma(t) d t \quad \text { for all } \gamma \in L^{2}(\mathbb{R}) \text {. }
$$

Then we say that $F$ is differentiable and we call $\psi(t)$ the stochastic gradient of $F$ (or the Hida-Malliavin derivative of $F$ ). We use the notation

$$
D_{t} F=\psi(t)
$$

for the stochastic gradient of $F$ at $t \in \mathbb{R}$.

Note that - in spite of the notation $-D_{t} F$ is not a derivative w.r.t. $t$ but (a kind of) derivative w.r.t. $\omega \in \Omega$. 
Example 2.17 Suppose $F(\omega)=\langle\omega, f\rangle=\int_{\mathbb{R}} f(s) d B(s)$ for some $f \in L^{2}(\mathbb{R})$. Then by linearity

$$
D_{\gamma} F(\omega)=\lim _{\varepsilon \rightarrow 0} \frac{1}{\varepsilon}[\langle\omega+\varepsilon \gamma, f\rangle-\langle\omega, f\rangle]=\langle\gamma, f\rangle=\int_{\mathbb{R}} f(t) \gamma(t) d t
$$

for all $\gamma \in L^{2}(\mathbb{R})$. We conclude that $F$ is differentiable and

$$
D_{t}\left(\int_{\mathbb{R}} f(s) d B(s)\right)=f(t) \quad \text { for a.a. } t \text {. }
$$

(Note that this is only valid for deterministic integrands $f$. See Theorem 2.22 for the general case.)

We note two useful chain rules for stochastic differentiation:

Theorem 2.18 (Chain rule I) Let $\phi: \mathbb{R}^{n} \rightarrow \mathbb{R}$ be a Lipschitz continuous function, i.e. there exists $C<\infty$ such that

$$
|\phi(x)-\phi(y)| \leq C|x-y| \quad \text { for all } x, y \in \mathbb{R}^{n} .
$$

Let $X=\left(X_{1}, \ldots, X_{n}\right)$ where each $X_{i}: \Omega \rightarrow \mathbb{R}$ is differentiable. Then $\phi(X)$ is differentiable and

$$
D_{t} \phi(X)=\sum_{k=1}^{n} \frac{\partial \phi}{\partial x_{k}}(X) D_{t} X_{k}
$$

We refer to [Nu1] for a proof.

If $f(x)=\sum_{m=0}^{\infty} a_{m} x^{m}$ is a real analytic function and $X \in(\mathcal{S})^{*}$ we put

$$
f^{\diamond}(X)=\sum_{m=0}^{\infty} a_{m} X^{\diamond m}
$$

provided the sum converges in $(\mathcal{S})^{*}$.

We call $f^{\diamond}(X)$ the Wick version of $f(X)$. A similar definition applies to real analytic functions on $\mathbb{R}^{n}$.

Theorem 2.19 (The Wick chain rule) Let $f: \mathbb{R}^{n} \rightarrow \mathbb{R}$ be real analytic and let $X=$ $\left(X_{1}, \ldots, X_{n}\right) \in\left((\mathcal{S})^{*}\right)^{n}$. Then if $f^{\diamond}(X) \in(\mathcal{S})^{*}$

$$
D_{t}\left(f^{\diamond}(X)\right)=\sum_{k=1}^{n}\left(\frac{\partial f}{\partial x_{k}}\right)^{\diamond}(X) \diamond D_{t} X_{k} ; \quad t \in \mathbb{R} .
$$


We refer to $[\mathrm{B} \emptyset \mathrm{SW}]$ for a proof.

Note that by Example 2.17 and the chain rule (2.47) we have

$$
D_{t} \mathcal{H}_{\alpha}(\omega)=\sum_{i=1}^{m} \alpha_{i} \mathcal{H}_{\alpha-\varepsilon^{(i)}}(\omega) \xi_{i}(t) \in(\mathcal{S})^{*} \quad \text { for all } t .
$$

In fact, using the topology for $(\mathcal{S})^{*}$ one can prove

Theorem 2.20 Let $F \in(\mathcal{S})^{*}$. Then $F$ is differentiable, and if $F$ has the expansion

$$
F(\omega)=\sum_{\alpha \in \mathcal{J}} c_{\alpha} \mathcal{H}_{\alpha}(\omega)
$$

then

$$
D_{t} F(\omega)=\sum_{\alpha, i} c_{\alpha} \alpha_{i} \mathcal{H}_{\alpha-\varepsilon^{(i)}}(\omega) \xi_{i}(t) \quad \text { for all } t \in \mathbb{R} .
$$

The stochastic gradient is the key to the connection between forward integrals and Skorohod integrals:

Theorem 2.21 Suppose $Y: \mathbb{R} \rightarrow(\mathcal{S})^{*}$ is caglad. Then

$$
\int_{0}^{T} Y(t) d^{-} B(t)=\int_{0}^{T} Y(t) \delta B(t)+\int_{0}^{T} D_{t^{+}} Y(t) d t \quad \text { for all } T>0,
$$

provided that the integrals exist, where

$$
D_{t^{+}} Y(t)=\lim _{s \rightarrow t^{+}} D_{s} Y(t) .
$$

We now mention without proofs some of the most fundamental results from stochastic differential and integral calculus. For proofs we refer to $[\mathrm{NP}]$ and $[\mathrm{B} \emptyset \mathrm{SW}]$.

\section{Theorem 2.22 (Fundamental theorem of stochastic calculus)}

Suppose $Y(\cdot): \mathbb{R} \rightarrow(\mathcal{S})^{*}$ and $D_{t} Y(\cdot): \mathbb{R} \rightarrow(\mathcal{S})^{*}$ are Skorohod integrable. Then

$$
D_{t}\left(\int_{\mathbb{R}} Y(s) \delta B(s)\right)=\int_{\mathbb{R}} D_{t} Y(s) \delta B(s)+Y(t) .
$$

Theorem 2.23 (Relation between the Wick product and the ordinary product) Suppose $g \in L^{2}(\mathbb{R})$ is deterministic and that $F \in L^{2}(P)$. Then

$$
F \diamond \int_{\mathbb{R}} g(t) d B(t)=F \cdot \int_{\mathbb{R}} g(t) d B(t)-\int_{\mathbb{R}} g(t) D_{t} F d t .
$$


Corollary 2.24 Let $g \in L^{2}(\mathbb{R})$ be deterministic and $F \in L^{2}(P)$. Then

$$
E\left[F \cdot \int_{\mathbb{R}} g(t) d B(t)\right]=E\left[\int_{\mathbb{R}} g(t) D_{t} F d t\right]
$$

provided that the integrals converge.

Theorem 2.25 (Integration by parts) Let $F \in L^{2}(P)$ and assume that $Y: \mathbb{R} \times \Omega \rightarrow \mathbb{R}$ is Skorohod integrable with $\int_{\mathbb{R}} Y(t) \delta B(t) \in L^{2}(P)$. Then

$$
F \int_{\mathbb{R}} Y(t) \delta B(t)=\int_{\mathbb{R}} F Y(t) \delta B(t)+\int_{\mathbb{R}} Y(t) D_{t} F d t
$$

provided that the integral on the extreme right converges in $L^{2}(P)$.

This immediately gives the following generalization of Corollary 2.24:

Corollary 2.26 Let $F$ and $Y(t)$ be as in Theorem 2.24. Then

$$
E\left[F \int_{\mathbb{R}} Y(t) \delta B(t)\right]=E\left[\int_{\mathbb{R}} Y(t) D_{t} F d t\right] .
$$

Theorem 2.27 (The Itô-Skorohod isometry) Suppose $Y: \mathbb{R} \times \Omega \rightarrow \mathbb{R}$ is Skorohod integrable with $\int_{\mathbb{R}} Y(t) \delta B(t) \in L^{2}(P)$. Then

$$
E\left[\left(\int_{\mathbb{R}} Y(t) \delta B(t)\right)^{2}\right]=E\left[\int_{\mathbb{R}} Y^{2}(t) d t\right]+E\left[\int_{\mathbb{R}} \int_{\mathbb{R}} D_{t} Y(s) D_{s} Y(t) d s d t\right] .
$$

Using Theorem 2.23 we obtain the following relation between forward integrals and Skorohod integrals:

Theorem 2.28 Suppose that $Y:[0, T] \rightarrow(\mathcal{S})^{*}$ is caglad and Skorohod integrable over $[0, T]$. Moreover, suppose that

$$
\int_{0}^{T} D_{t^{+}} Y(t) d t \quad \text { exists }
$$

where

$$
D_{t^{+}} Y(t)=\lim _{s \rightarrow t^{+}} D_{s} Y(t) .
$$

Then

$$
\int_{0}^{T} Y(t) d^{-} B(t)=\int_{0}^{T} Y(t) \delta B(t)+\int_{0}^{T} D_{t^{+}} Y(t) d t
$$




\section{$3 \quad$ Fractional stochastic calculus}

We now consider the corresponding calculus for fractional Brownian motion $B_{H}(t)$ with arbitrary Hurst parameter $H \in(0,1)$. It turns out that it is possible to transform the calculus for $B(t)$ into the calculus for $B_{H}(t)$ by means of an operator $M$. This is the idea of $[\mathrm{EvdH}]$, which we now describe. The approach of $[\mathrm{EvdH}]$ represents an extension to all $H \in(0,1)$ of the fractional white noise calculus for $H \in\left(\frac{1}{2}, 1\right)$ introduced by $[\mathrm{H} \varnothing]$. For details we refer to $[\mathrm{EvdH}],[\mathrm{H} \varnothing],[\mathrm{B} \varnothing \mathrm{SW}]$ and $[\mathrm{BH} \varnothing \mathrm{Z}]$. See also [DÜ] and [Nu2] for an alternative approach.

Definition 3.1 For $H \in(0,1)$ put

$$
c_{H}=\left[2 \Gamma\left(H-\frac{1}{2}\right) \cos \left(\frac{\pi}{2}\left(H-\frac{1}{2}\right)\right)\right]^{-1}[\Gamma(2 H+1) \sin (\pi H)]^{1 / 2}
$$

where $\Gamma(\cdot)$ is the Gamma function. Define the operator $\mathrm{M}=\mathrm{M}_{H}$ on $\mathcal{S}(\mathbb{R})$ by

$$
\widehat{\mathrm{Mf}}(y)=c_{H}|y|^{\frac{1}{2}-H} \hat{f}(y) ; \quad f \in \mathcal{S}(\mathbb{R}),
$$

where in general

$$
\hat{g}(y)=\frac{1}{\sqrt{2 \pi}} \int_{\mathbb{R}} e^{-i x y} g(x) d x
$$

is the Fourier transform of $g$.

Let $L_{H}^{2}(\mathbb{R})$ be the closure of $\mathcal{S}(\mathbb{R})$ in the norm

$$
\|f\|_{L_{H}^{2}(\mathbb{R})}^{2}=(\mathrm{M} f, \mathrm{M} f)_{L^{2}(\mathbb{R})}=\int_{\mathbb{R}}(\mathrm{M} f(x))^{2} d x ; \quad f \in \mathcal{S}(\mathbb{R}) .
$$

Then the operator $\mathrm{M}$ extends in a natural way to an isometry between the two Hilbert spaces $L^{2}(\mathbb{R})$ and $L_{H}^{2}(\mathbb{R})$. Note that

$$
(\widehat{\mathrm{M} f}, \widehat{\mathrm{M} g})=(\mathrm{M} f, \mathrm{M} g)=\left(f, \mathrm{M}^{2} g\right) \quad \text { for } f, g \in L_{H}^{2}(\mathbb{R})
$$

Now define

$$
\tilde{B}_{H}(t)=\tilde{B}_{H}(t, \omega)=\left\langle\omega, \mathrm{M} \mathcal{X}_{[0, t]}\right\rangle .
$$

Then by Section 2 we see that $\tilde{B}(t)$ is a Gaussian process with mean 0 and covariance

$$
\begin{aligned}
& E\left[\tilde{B}_{H}(s) \tilde{B}_{H}(t)\right]=\left(\mathrm{M} \mathcal{X}_{[0, s]}, \mathrm{M} \mathcal{X}_{[0, t]}\right) \\
& =\left(\mathcal{X}_{[0, s]}, \mathcal{X}_{[0, t]}\right)_{L_{H}^{2}(\mathbb{R})}=\frac{1}{2}\left(|s|^{2 H}+|t|^{2 H}-|s-t|^{2 H}\right),
\end{aligned}
$$

by (A.10) in $[\mathrm{EvdH}]$.

Therefore $\tilde{B}_{H}(t)$ has a continuous version, denoted by $B_{H}(t)$, which is a fractional Brownian motion with Hurst coefficient $H$. Arguing as in Section 2 we see that if

$$
f(t)=\sum_{j} a_{j} \mathcal{X}_{\left[t_{j}, t_{j+1}\right)}(t)
$$


is a (deterministic) step function, then

$$
\langle\omega, \mathrm{M} f\rangle=\sum_{j} a_{j}\left(B_{H}\left(t_{j+1}\right)-B_{H}\left(t_{j}\right)\right)=\int_{\mathbb{R}} f(t) d B_{H}(t) .
$$

On the other hand, we know that

$$
\langle\omega, \mathrm{M} f\rangle=\int_{\mathbb{R}} \mathrm{M} f(t) d B(t) .
$$

Therefore

$$
\int_{\mathbb{R}} f(t) d B_{H}(t)=\int_{\mathbb{R}} \mathrm{M} f(t) d B(t)
$$

for all step functions $f$, and hence for all $f \in L_{H}^{2}(\mathbb{R})$.

The chaos expansion of $B_{H}(t) \in L^{2}(P)$ is

$$
\begin{aligned}
B_{H}(t) & =\left\langle\omega, \mathrm{M} \mathcal{X}_{[0, t]}\right\rangle=\left\langle\omega, \sum_{k=1}^{\infty}\left(\mathrm{M} \mathcal{X}_{[0, t]}, \xi_{k}\right) \xi_{k}\right\rangle \\
& =\sum_{k=1}^{\infty}\left(\mathcal{X}_{[0, t]}, \mathrm{M} \xi_{k}\right)\left\langle\omega, \xi_{k}\right\rangle=\sum_{k=1}^{\infty} \int_{0}^{t} \mathrm{M} \xi_{k}(s) d s \mathcal{H}_{\varepsilon(k)}(\omega) .
\end{aligned}
$$

Therefore, if we define fractional white noise $W_{H}(t)$ by

$$
W_{H}(t)=\sum_{k=1}^{\infty} \mathrm{M} \xi_{k}(t) \mathcal{H}_{\varepsilon(k)}(\omega)
$$

then $W_{H}(t) \in(\mathcal{S})^{*}$ and

$$
\frac{d B_{H}(t)}{d t}=W_{H}(t) \quad \text { in }(\mathcal{S})^{*} .
$$

In view of this and Theorem 2.9 the following definition is natural:

Definition 3.2 The Skorohod integral of a function $Y: \mathbb{R} \rightarrow(\mathcal{S})^{*}$ with respect to $B_{H}(t)$ is defined by

$$
\int_{\mathbb{R}} Y(t) \delta B_{H}(t)=\int_{\mathbb{R}} Y(t) \diamond W_{H}(t) d t
$$

provided that $Y(t) \diamond W_{H}(t)$ is integrable in $(\mathcal{S})^{*}$. 
We can in a natural way extend the M-operator to functions $Y: \mathbb{R} \rightarrow(\mathcal{S})^{*}$ whose chaos expansion

$$
Y(t)=\sum_{q \in \mathcal{J}} c_{\alpha}(t) \mathcal{H}_{\alpha}(\omega)
$$

has coefficients $c_{\alpha} \in L_{H}^{2}(\mathbb{R})$, as follows:

$$
\mathrm{M} Y(t)=\sum_{\alpha \in \mathcal{J}} \mathrm{M} c_{\alpha}(t) \mathcal{H}_{\alpha}(\omega)
$$

This is well-defined if the series converges in $(\mathcal{S})^{*}$. With this extension of $\mathrm{M}$ we note that the connection between the classical white noise $W(t)$ and the fractional white noise $W_{H}(t)$ can be written

$$
W_{H}(t)=\mathrm{M} W(t) ; \quad t \in \mathbb{R} .
$$

Combining this with Definition 3.2 we get

Theorem 3.3 Let $Y: \mathbb{R} \rightarrow(\mathcal{S})^{*}$. Suppose $Y(t)$ has the expansion

$$
Y(t)=\sum_{\alpha \in \mathcal{J}} c_{\alpha}(t) \mathcal{H}_{\alpha}(\omega) ; \quad t \in \mathbb{R}
$$

where

$$
c_{\alpha}(\cdot) \in L_{H}^{2}(\mathbb{R}) \quad \text { for all } \alpha \in \mathcal{J} \text {. }
$$

Then

$$
\int_{\mathbb{R}} Y(t) \delta B_{H}(t)=\sum_{\substack{\alpha \in \mathcal{J} \\ k \in \mathbb{N}}}\left(c_{\alpha}, e_{k}\right)_{L_{H}^{2}(\mathbb{R})} \mathcal{H}_{\alpha+\varepsilon^{(k)}}(\omega),
$$

provided that the right hand side converges in $(\mathcal{S})^{*}$.

Note in particular that if $\int_{\mathbb{R}} Y(t) \delta B_{H}(t) \in L^{2}(P)$ then

$$
E\left[\int_{\mathbb{R}} Y(t) \delta B_{H}(t)\right]=0 .
$$

Proof.

$$
\begin{aligned}
\int_{\mathbb{R}} Y(t) & \delta B_{H}(t)=\int_{\mathbb{R}} Y(t) \diamond W_{H}(t) d t \\
& =\int_{\mathbb{R}} Y(t) \diamond \sum_{k=1}^{\infty} \mathrm{M} \xi_{k}(t) \mathcal{H}_{\varepsilon^{(k)}}(\omega) d t \\
& =\sum_{\alpha, k}\left(c_{\alpha}, \mathrm{M} \xi_{k}\right) \mathcal{H}_{\alpha+\varepsilon^{(k)}}(\omega)=\sum_{\alpha, k}\left(\mathrm{M} c_{\alpha}, \xi_{k}\right) \mathcal{H}_{\alpha+\varepsilon^{(k)}}(\omega) \\
& =\sum_{\alpha, k}\left(c_{\alpha}, e_{k}\right)_{L_{H}^{2}(\mathbb{R})} \mathcal{H}_{\alpha+\varepsilon^{(k)}}(\omega) .
\end{aligned}
$$


We also note the following relation between the Skorohod integrals w.r.t. $B_{H}(\cdot)$ and $B(\cdot)$ :

$$
\int_{\mathbb{R}} Y(s) \delta B_{H}(s)=\int_{\mathbb{R}} \mathrm{M}_{s} Y(s) \delta B(s),
$$

where $\mathrm{M}_{s}$ indicates that $\mathrm{M}$ is operating on the variable $s$. This follows from (3.15) and Theorem 2.12 .

Example 3.4 What is $\int_{0}^{T} B_{H}(t) \delta B_{H}(t)$ ? We can answer this by using Wick calculus as in Example 2.10:

$$
\begin{gathered}
\int_{0}^{T} B_{H}(t) \delta B_{H}(t)=\int_{0}^{T} B_{H}(t) \diamond W_{H}(t) d t=\int_{0}^{T} B_{H}(t) \diamond \frac{d}{d t} B_{H}(t) d t \\
=\left.\frac{1}{2}\right|_{0} ^{T} B_{H}^{\diamond 2}(t)=\frac{1}{2} B_{H}^{\diamond 2}(T)=\frac{1}{2} B_{H}^{2}(T)-\frac{1}{2} T^{2 H},
\end{gathered}
$$

because, by (3.5) and (2.30),

$$
\begin{aligned}
B_{H}^{\diamond 2}(T) & =\left\langle\omega, \mathrm{M} \mathcal{X}_{[0, T]}\right\rangle \diamond\left\langle\omega, \mathrm{M} \mathcal{X}_{[0, T]}\right\rangle \\
& =\left\langle\omega, \mathrm{M} \mathcal{X}_{[0, T]}\right\rangle \cdot\left\langle\omega, \mathrm{M} \mathcal{X}_{[0, T]}\right\rangle-\left(\mathrm{M} \mathcal{X}_{[0, T]}, \mathrm{M} \mathcal{X}_{[0, T]}\right) \\
& =B_{H}(T) \cdot B_{H}(T)-\left(\mathcal{X}_{[0, T]}, \mathcal{X}_{[0, T]}\right)_{L_{H}^{2}(\mathbb{R})} \\
& =B_{H}^{2}(T)-T^{2 H} \quad(\text { by }(\mathrm{A} .10) \text { in }[\operatorname{EvdH}]) .
\end{aligned}
$$

This result could also have been deduced from the following version of the Itô formula.

Theorem 3.5 [BØSW, Theorem 3.8] (Itô formula for fractional Skorohod integrals) Let $f(s, x): \mathbb{R} \times \mathbb{R} \rightarrow \mathbb{R}$ belong to $C^{1,2}(\mathbb{R} \times \mathbb{R})$ and assume that the three random variables

$$
f\left(t, B_{H}(t)(t)\right), \quad \int_{0}^{t} \frac{\partial f}{\partial s}\left(s, B_{H}(s)\right) d s \quad \text { and } \quad \int_{0}^{t} \frac{\partial^{2} f}{\partial x^{2}}\left(s, B_{H}(s)\right) s^{2 H-1} d s
$$

all belong to $L^{2}(P)$. Then

$$
\begin{aligned}
f\left(t, B_{H}(t)(t)\right) & =f(0,0)+\int_{0}^{t} \frac{\partial f}{\partial s}\left(s, B_{H}(s)\right) d s \\
+ & \int_{0}^{t} \frac{\partial f}{\partial x}\left(s, B_{H}(s)\right) d B_{H}(s)+H \int_{0}^{t} \frac{\partial^{2} f}{\partial x^{2}}\left(s, B_{H}(s)\right) s^{2 H-1} d s .
\end{aligned}
$$

Proof. There are several versions of this result. See $[\mathrm{B}],[\mathrm{Mi}],[\mathrm{vdH}]$ and $[\mathrm{B} \emptyset \mathrm{SW}]$. This result is valid for all $H \in(0,1)$, but if we restrict ourselves to $\frac{1}{2}<H<1$ there is a more general Itô formula in $[\mathrm{DHP}]$ and $[\mathrm{B} \varnothing]$. 
Example 3.6 Let $\alpha, \beta \neq 0$ be constants. The fractional Skorohod equation

$$
\delta Y(t)=\alpha Y(t) d t+\beta Y(t) \delta B_{H}(t) ; \quad Y(0)>0
$$

i.e.

$$
Y(t)=Y(0)+\int_{0}^{t} \alpha Y(s) d s+\int_{0}^{t} \beta Y(s) \delta B_{H}(s) ; \quad t \geq 0
$$

has the unique solution

$$
Y(t)=Y(0) \exp \left(\beta B_{H}(t)+\alpha t-\frac{1}{2} \beta^{2} t^{2 H}\right) ; \quad t>0 .
$$

This follows by applying Theorem 3.5 to the process

$$
X(t)=\alpha t-\frac{1}{2} \beta^{2} t^{2 H}+\beta B_{H}(t)
$$

and the function

$$
f(x)=Y(0) \exp x .
$$

In analogy with the classical case we call this process $Y(t)$ the geometric Skorohod fractional Brownian motion. Note that if we put $H=\frac{1}{2}$ we get the classical geometric Brownian motion.

We proceed to consider differentiation:

Definition 3.7 The Hida-Malliavin derivative $D_{t}^{(H)}$ (or stochastic gradient) of an element $F \in(\mathcal{S})^{*}$ is defined by

$$
D_{t}^{(H)} F=\mathrm{M}^{-1} D_{t} F ; \quad t \in \mathbb{R} .
$$

By Theorem 2.20 we see that if $F$ has the expansion

$$
F(\omega)=\sum_{\alpha \in \mathcal{J}} c_{\alpha} \mathcal{H}_{\alpha}(\omega)
$$

then

$$
D_{t}^{(H)} F=\sum_{\substack{\alpha \in \mathcal{J} \\ i \in \mathbb{N}}} c_{\alpha} \alpha_{i} \mathcal{H}_{\alpha-\varepsilon^{(i)}}(\omega) e_{i}(t) ; \quad t \in \mathbb{R}
$$

We can now formulate the fractional analogue of Theorem 2.22:

Theorem 3.8 [BØSW, Theorem 5.3] (Fractional fundamental theorem of calculus) Suppose $Y(\cdot): \mathbb{R} \rightarrow(\mathcal{S})^{*}$ and $D_{t}^{(H)} Y(\cdot): \mathbb{R} \rightarrow(\mathcal{S})^{*}$ are Skorohod integrable w.r.t. $B_{H}$. Then

$$
D_{t}^{(H)}\left(\int_{\mathbb{R}} Y(s) \delta B_{H}(s)\right)=\int_{\mathbb{R}} D_{t}^{(H)} Y(s) \delta B_{H}(s)+Y(t) .
$$


Proof. By (3.22), (3.16) and Theorem 2.22 we get

$$
\begin{aligned}
D_{t}^{(H)}\left(\int_{\mathbb{R}} Y\right. & \left.(s) \delta B_{H}(s)\right)=\mathrm{M}_{t}^{-1} D_{t}\left(\int_{\mathbb{R}} \mathrm{M}_{s} Y(s) \delta B(s)\right) \\
& =\mathrm{M}_{t}^{-1} \int_{\mathbb{R}} D_{t}\left(\mathrm{M}_{s} Y(s)\right) \delta B(s)+\mathrm{M}_{t}^{-1} \mathrm{M}_{t} Y(t) \\
& =\int_{\mathbb{R}} \mathrm{M}_{t}^{-1} D_{t}\left(\mathrm{M}_{s} Y(s)\right) \delta B(s)+Y(t) \\
& =\int_{\mathbb{R}} D_{t}^{(H)}\left(\mathrm{M}_{s} Y(s)\right) \delta B(s)+Y(t) \\
& =\int_{\mathbb{R}} \mathrm{M}_{s}\left(D_{t}^{(H)} Y(s)\right) \delta B(s)+Y(t) \\
& =\int_{\mathbb{R}} D_{t}^{(H)} Y(s) \delta B_{H}(s)+Y(t) .
\end{aligned}
$$

Theorem 3.9 [BØSW, Theorem 5.4] (Fractional integration by parts) Let $F \in$ $L^{2}(P)$ and assume that $Y: \mathbb{R} \times \Omega \rightarrow \mathbb{R}$ is Skorohod integrable w.r.t. $B_{H}$ with $\int_{\mathbb{R}} Y(t) \delta B_{H}(t) \in$ $L^{2}(P)$. Then

$$
F \int_{\mathbb{R}} Y(t) \delta B_{H}(t)=\int_{\mathbb{R}} F Y(t) \delta B_{H}(t)+\int_{\mathbb{R}} Y(t) \mathrm{M}_{t}^{2} D_{t}^{(H)} F d t .
$$

Proof. By (3.16), Theorem 2.25 and (3.22) we get

$$
\begin{gathered}
F \int_{\mathbb{R}} Y(t) \delta B_{H}(t)=F \int_{\mathbb{R}} \mathrm{M}_{t} Y(t) \delta B(t)=\int_{\mathbb{R}} F \mathrm{M}_{t} Y(t) \delta B(t)+\int_{\mathbb{R}} \mathrm{M}_{t} Y(t) D_{t} F d t \\
=\int_{\mathbb{R}} \mathrm{M}_{t}(F Y(t)) \delta B(t)+\int_{\mathbb{R}} \mathrm{M}_{t} Y(t) \mathrm{M}_{t} D_{t}^{(H)} F d t \\
=\int_{\mathbb{R}} F Y(t) \delta B_{H}(t)+\int_{\mathbb{R}} Y(t) \mathrm{M}_{t}^{2} D_{t}^{(H)} F d t .
\end{gathered}
$$

Corollary 3.10 Let $F$ and $Y(t)$ be as in Theorem 3.9. Then

$$
E\left[F \int_{\mathbb{R}} Y(t) \delta B_{H}(t)\right]=E\left[\int_{\mathbb{R}} Y(t) \mathrm{M}_{t}^{2} D_{t}^{(H)} F d t\right] .
$$

We also note the following fractional version of Theorem 2.27: 
Theorem 3.11 (The fractional Itô-Skorohod isometry [EvdH]) Suppose

$Y: \mathbb{R} \times \Omega \rightarrow \mathbb{R}$ is Skorohod-integrable with respect to $B_{H}$ with $\int_{\mathbb{R}} Y(t) \delta B_{H}(t) \in L^{2}(P)$. Then

$$
\begin{aligned}
& E\left[\left(\int_{\mathbb{R}} Y(t) \delta B_{H}(t)\right)^{2}\right] \\
& \quad=E\left[\int_{\mathbb{R}}(\mathrm{M} Y(t))^{2} d t\right]+E\left[\int_{\mathbb{R}} \int_{\mathbb{R}} D_{t}^{(H)} \mathrm{M}_{s}^{2} Y(s) \cdot D_{s}^{(H)} \mathrm{M}_{t}^{2} Y(t) d s d t\right] .
\end{aligned}
$$

Proof. This follows by combining Theorem 2.27 with (3.16) and (3.22). We omit the details.

Finally we turn to the fractional forward integral. This is defined in the same way as in the classical case (Definition 2.13):

Definition 3.12 The forward integral of a function $Y: \mathbb{R} \rightarrow(\mathcal{S})^{*}$ with respect to $B_{H}(t)$ is defined by:

$$
\int_{\mathbb{R}} Y(t) d^{-} B_{H}(t)=\lim _{\varepsilon \rightarrow 0} \int_{\mathbb{R}} Y(t) \frac{B_{H}(t+\varepsilon)-B_{H}(t)}{\varepsilon} d t,
$$

provided that the limit exists in $(\mathcal{S})^{*}$.

Just as in Theorem 2.14 we have

Theorem 3.13 Suppose $Y:[0, T] \rightarrow(\mathcal{S})^{*}$ is caglad and forward integrable over $[0, T]$ w.r.t. $B_{H}(\cdot)$. Then

$$
\int_{0}^{T} Y(t) d^{-} B(t)=\lim _{\Delta t_{j} \rightarrow 0} \sum_{j=0}^{N-1} Y\left(t_{j}\right) \cdot\left(B_{H}\left(t_{j+1}\right)-B_{H}\left(t_{j}\right)\right)
$$

$\left(\right.$ limit in $\left.(\mathcal{S})^{*}\right)$.

Remark 3.14 In the special case when $Y=Y(t, \omega):[0, T] \times \Omega \rightarrow \mathbb{R}$ is a classical stochastic process (and $Y(t, \cdot) \in(\mathcal{S})^{*}$ for all $t$ ) and the limit in (3.29) exists for a.a. $\omega$, the forward integral of $Y$ coincides with the pathwise integral (or more precisely the left Young (LY) integral of $Y$ ) with respect to $d B_{H}(t)$. See [N] for details.

Definition 3.15 A function $Y:[0, T] \rightarrow(\mathcal{S})^{*}$ with expansion

$$
Y(t)=\sum_{\alpha \in \mathcal{J}} c_{\alpha}(t) \mathcal{H}_{\alpha}(\omega)
$$

belongs to the space $\mathbb{D}_{1,2}^{(H)}$ if

$$
\|Y\|_{\mathbb{D}_{1,2}^{(H)}}^{2}:=\sum_{\alpha \in \mathcal{J}} \sum_{i=1}^{\infty} \alpha_{i} \alpha !\left(c_{\alpha}, \xi_{i}\right)^{2}<\infty
$$


where

$$
\left(c_{\alpha}, \xi_{i}\right)=\int_{0}^{T} c_{\alpha}(s) \xi_{i}(s) d s .
$$

The analogue of Theorem 2.28 is the following:

Theorem 3.16 Suppose that $Y:[0, T] \rightarrow(\mathcal{S})^{*}$ is cadlag and Skorohod integrable over $[0, T]$ w.r.t. $B_{H}(t)$. Moreover, suppose that $Y \in \mathbb{D}_{1,2}^{(H)}$. Then

$$
\int_{0}^{T}\left[\mathrm{M}_{t}^{2} D_{t}^{(H)} Y(u)\right]_{u=t} d t \quad \text { exists in } L^{2}(P)
$$

and

$$
\int_{0}^{T} Y(t) d^{-} B_{H}(t)=\int_{0}^{T} Y(t) \delta B_{H}(t)+\int_{0}^{T}\left[\mathrm{M}_{t}^{2} D_{t}^{(H)} Y(u)\right]_{u=t} d t .
$$

Proof. We refer to $[\mathrm{B} \varnothing]$ for details. See also $[\mathrm{Mi}]$.

We end this section by giving an Itô formula for forward integrals w.r.t. fractional Brownian motion:

A forward fractional Itô process is a process of the form

$$
X(t)=x+\int_{0}^{t} u(s, \omega) d s+\int_{0}^{t} v(s, \omega) d^{-} B_{H}(s) ; \quad t \geq 0
$$

where $u(s, \omega)$ and $v(s, \omega)$ are realvalued, measurable (not necessarily adapted) processes such that

$$
\int_{0}^{t}|u(s, \omega)| d s<\infty \quad \text { and } \quad \int_{0}^{t} v(s, \omega) d^{-} B_{H}(s) \quad \text { exists a.e.. }
$$

In this case we use the shorthand notation

$$
d^{-} X(t)=u(t) d t+v(t) d^{-} B_{H}(t) ; \quad X(0)=x .
$$

Theorem 3.17 (An Itô formula for forward fractional processes) Suppose

$$
H \in\left(\frac{1}{2}, 1\right) \text {. }
$$

Let $f \in C^{1}(\mathbb{R})$ and put

$$
Y(t)=f(X(t))
$$

where $X(t)$ is given by (3.32). Then

$$
d^{-} Y(t)=f^{\prime}(X(t)) d^{-} X(t) .
$$


Proof. This is a classic result about forward (pathwise) integration. A direct proof can be found in $[\mathrm{B} \varnothing]$. See also $[\mathrm{N}],[\mathrm{Nu} 2]$ and $[\mathrm{RV}]$ and the references therein. If $f$ posseses higher order regularity then a corresponding (but more complicated) Itô formula can be obtained for lower values of $H$. See e.g. [CQ] and [GNRV].

Example 3.18 The fractional forward equation

$$
d^{-} X(t)=\alpha X(t) d t+\beta X(t) d^{-} B_{H}(t) ; \quad X(0)=x>0
$$

has for $\frac{1}{2}<H<1$ the unique solution

$$
X(t)=x \exp \left(\beta B_{H}(t)+\alpha t\right) ; \quad t \geq 0 .
$$

\section{Fractional Brownian motion in finance}

We now use the mathematical machinery described in the earlier sections to study finance models involving $f \mathrm{Bm}$. We have seen that there are two natural ways of defining integration with respect to $f B m$ :

(a) The pathwise (forward) integration

(b) The Skorohod integration.

Therefore we discuss these two cases separately:

\section{a) The pathwise integration model $\left(\frac{1}{2}<H<1\right)$}

For simplicity we concentrate on the simplest nontrivial type of market, namely on the $f B m$ version of the classical Black-Scholes market, as follows:

Suppose there are two investment possibilities:

(i) A safe or risk free investment, with price dynamics

$$
d S_{0}(t)=r S_{0}(t) d t ; \quad S_{0}(0)=1
$$

and

(ii) a risky investment, with price dynamics

$$
d^{-} S_{1}(t)=\mu S_{1}(t) d t+\sigma S_{1}(t) d^{-} B_{H}(t) ; \quad S_{1}(0)=x>0,
$$

where $r, \mu, \sigma \neq 0$ and $x>0$ are constants. By Example 3.17 we know that the solution of this equation is

$$
S_{1}(t)=x \exp \left(\sigma B_{H}(t)+\mu t\right) ; \quad t \geq 0 .
$$

Let $\left\{\mathcal{F}_{t}^{H}\right\}_{t \geq 0}$ be the filtration of $B_{H}(\cdot)$, i.e. $\mathcal{F}_{t}^{H}$ is the $\sigma$-algebra generated by the random variables $B_{H}(s), s \leq t$. 
A portfolio in this market is a 2-dimensional $\mathcal{F}_{t}^{H}$-adapted stochastic proces $\theta(t)=$ $\left(\theta_{0}(t), \theta_{1}(t)\right)$ where $\theta_{i}(t)$ gives the number of units of investment number $i$ held at time $t, i=0,1$. The corresponding wealth process $V^{\theta}(t)$ is defined by

$$
V^{\theta}(t)=\theta(t) \cdot S(t)=\theta_{0}(s) S_{0}(t)+\theta_{1}(t) S_{1}(t),
$$

where

$$
S(t)=\left(S_{0}(t), S_{0}(t)\right)
$$

We say that $\theta$ is pathwise self-financing if

$$
d^{-} V^{\theta}(t)=\theta(t) \cdot d^{-} S(t)
$$

i.e.

$$
V^{\theta}(t)=V^{\theta}(0)+\int_{0}^{t} \theta_{0}(s) d S_{0}(s)+\int_{0}^{t} \theta_{1}(s) d^{-} S_{1}(s)
$$

If, in addition, $V^{\theta}(t)$ is lower bounded, then we call the portfolio $\theta$ (pathwise) admissible.

Definition 4.1 A pathwise admissible portfolio $\theta$ is called an arbitrage if the corresponding wealth process $V^{\theta}(t)$ satisfies thee following 3 conditions:

$$
\begin{aligned}
& V^{\theta}=0 \\
& V^{\theta}(T) \geq 0 \quad \text { a.s. } \\
& P\left[V^{\theta}(T)>0\right]>0 .
\end{aligned}
$$

Remark 4.2 The non-existence of arbitrage in a market is a basic equilibrium condition. It is not possible to make a sensible mathematical theory for a market with arbitrage. Therefore one of the first things to check in a mathematical finance model is whether arbitrages exist. In the above pathwise $f B m$ market the existence of arbitrage was proved by Rogers $[R]$ in 1997. Subsequently several simple examples of arbitrage were found. See e.g. [D], [Sa], [Sh]. Note, however, that the existence of arbitrage in this pathwise model is already a direct consequence of Theorem 7.2 in [DS] (1994): There it is proved in general that if there is no arbitrage using simple portfolios (with pathwise products), then the price process is a semimartingale. Hence, since the process $S_{1}(t)$ given by (4.2) is not a semimartingale, an arbitrage must exist.

Here is a simple arbitrage example, due to [D] and [Sh]:

For simplicity assume that

$$
\mu=r \quad \text { and } \quad \sigma=x=1 .
$$

Define

$$
\theta_{0}(t)=1-\exp \left(2 B_{H}(t)\right), \quad \theta_{1}(t)=2\left(\exp \left(B_{H}(t)\right)-1\right) .
$$


Then the corresponding wealth process is

$$
\begin{aligned}
V^{\theta}(t) & =\theta_{0}(t) S_{0}(t)+\theta_{1}(t) S_{1}(t) \\
& =\left(1-\exp \left(2 B_{H}(t)\right)\right) \exp (r t)+2\left(\exp \left(B_{H}(t)\right)-1\right) \exp \left(B_{H}(t)+r t\right) \\
& =\exp (r t)\left(\exp \left(B_{H}(t)\right)-1\right)^{2}>0 \quad \text { for a.a. }(t, \omega) .
\end{aligned}
$$

This portfolio is self-financing, since

$$
\begin{aligned}
\theta_{0}(t) & d S_{0}(t)+\theta_{1}(t) d^{-} S_{1}(t) \\
& =\left(1-\exp \left(2 B_{H}(t)\right)\right) r \exp (r t) d t+2\left(\exp \left(B_{H}(t)\right)-1\right) S_{1}(t)\left[r d t+d^{-} B_{H}(t)\right] \\
& =r \exp (r t)\left(\exp \left(B_{H}(t)\right)-1\right)^{2} d t+2 \exp (r t)\left(\exp \left(B_{H}(t)\right)-1\right) \exp \left(B_{H}(t)\right) d^{-} B_{H}(t) \\
& =d\left(\exp (r t)\left(\exp \left(B_{H}(t)\right)-1\right)^{2}\right)=d^{-} V^{\theta}(t) .
\end{aligned}
$$

We have proved:

Theorem 4.3 ([D], [Sh]) The portfolio $\theta(t)=\left(\theta_{0}(t), \theta_{1}(t)\right)$ given by (4.11) is a (pathwise) arbitrage in the (pathwise) fractional Black-Scholes market given by (4.1), (4.2) and (4.10).

In view of this result the pathwise $f B m$ model is not suitable in finance, at least not in this simple form (but possibly in combination with classical Brownian motion).

\section{b) The Wick-Skorohod integration model $(0<H<1)$}

We now consider the Wick-Skorohod integration version of the market (4.1)-(4.2). Mathematically the model below is an extension to $H \in(0,1)$ of the model introduced in [HØ] for $H \in\left(\frac{1}{2}, 1\right)$. (Subsequently a related model, also valid for all $H \in(0,1)$, was presented in $[\mathrm{EvdH}]$.) However, compared to [HØ] we give a different interpretation of the mathematical concepts involved:

Assume that the values $S_{0}(t), S_{1}(t)$ of the risk free (e.g. bond) and risky asset (e.g. stock), respectively, are given by

$$
\text { (bond) } \quad d S_{0}(t)=r S_{0}(t) d t ; \quad S_{0}(0)=1
$$

and

$$
\text { (stock) } \quad \delta S_{1}(t)=\mu S_{1}(t) d t+\sigma S_{1}(t) \delta B_{H}(t) ; \quad S_{1}(0)=x>0
$$

where $r, \mu, \sigma \neq 0$ and $x>0$ are constants.

By Example 3.6 the solution of equation (4.14) is

$$
S_{1}(t)=x \exp \left(\sigma B_{H}(t)+\mu t-\frac{1}{2} \sigma^{2} t^{2 H}\right) ; \quad t \geq 0 .
$$

In this Wick-Skorohod model $S_{1}(t)$ does not represent the observed stock price at time $t$, but we give it a different interpretation: We assume that $S_{1}(t)$ represents in a broad sense the total value of the company and that it is not observed directly. Instead we adopt a quantum mechanical point of view, regarding $S_{1}(t, \omega)$ as a stochastic distribution in $\omega$ (represented 
mathematically as an element of $\left.(\mathcal{S})^{*}\right)$, and regarding the actual observed stock price $\hat{S}(t)$ as the result of applying $S_{1}(t, \cdot) \in(\mathcal{S})^{*}$ to a stochastic test function $\psi(\cdot) \in(\mathcal{S})$.

In other words,

$$
\hat{S}(t):=\langle S(t, \cdot), \psi(\cdot)\rangle=\langle S(t), \psi\rangle
$$

where in general $\langle F, \psi\rangle$ denotes the action of a stochastic distribution $F \in(\mathcal{S})^{*}$ to a stochastic test function $\psi \in(\mathcal{S})$. (See Section 2.)

We call such stochastic test functions $\psi$ market observers. We will assume that they have the form

$$
\begin{aligned}
\psi(\omega) & =\exp ^{\diamond}\left(\int_{\mathbb{R}} h(t) d B_{H}(t)\right) \\
& =\exp \left(\int_{\mathbb{R}} h(t) d B_{H}(t)-\frac{1}{2}\|h\|_{L_{H}^{2}(\mathbb{R})}^{2}\right) \quad \text { for some } h \in L_{H}^{2}(\mathbb{R}) .
\end{aligned}
$$

The set of all linear combinations of such $\psi$ is dense in both $(\mathcal{S})$ and $(\mathcal{S})^{*}$. Moreover, these $\psi$ are normalized, in the sense that

$$
E\left[\exp ^{\diamond}\left(\int_{\mathbb{R}} h(t) d B_{H}(t)\right)\right]=1 \quad \text { for all } h \in L_{H}^{2}(\mathbb{R}) .
$$

We let $\mathcal{D}$ denote the set of all market observers of the form (4.17).

Similarly, a generalized portfolio is another adapted process

$$
\theta(t)=\theta(t, \omega)=\left(\theta_{0}(t, \omega), \theta_{1}(t, \omega)\right) ; \quad(t, \omega) \in[0, T] \times \Omega
$$

representing a general strategy for choosing the number of units of investment number $i$ at time $t ; i=0,1$. (For example, $\theta_{1}(t)$ could be the usual "buy and hold" strategy, consisting of buying a certain number of stocks at a stopping time $\tau_{1}(\omega)$ and holding them until another stopping time $\tau_{2}(\omega)>\tau_{1}(\omega)$. Or $\theta_{1}(t)$ could be the strategy to hold a fixed fraction of the current wealth in stocks.) If the actual observed price at time $t$ is $\hat{S}_{1}(t)=\left\langle S_{1}(t, \cdot), \psi(\cdot)\right\rangle$, the actual number of stocks held is

$$
\hat{\theta}_{1}(t):=\left\langle\theta_{1}(t, \cdot), \psi(\cdot)\right\rangle .
$$

Thus the actual observed wealth $\hat{V}_{1}(t)$ held in the risky asset corresponding to this portfolio is

$$
\hat{V}_{1}(t)=\left\langle\theta_{1}(t), \psi\right\rangle \cdot\left\langle S_{1}(t), \psi\right\rangle .
$$

By Lemma 4.4 below this can be written

$$
\hat{V}_{1}(t)=\left\langle\theta_{1}(t) \diamond S_{1}(t), \psi\right\rangle,
$$

where $\diamond$ denotes the Wick product. In fact, $F:=\theta_{1}(t) \diamond S_{1}(t)$ is the unique $F \in(S)^{*}$ such that

$$
\langle F, \psi\rangle=\left\langle\theta_{1}(t), \psi\right\rangle \cdot\left\langle S_{1}(t), \psi\right\rangle \quad \text { for all } \psi \in \mathcal{D}
$$


In view of this it is natural to define the generalized total wealth process $V(t, \omega)$ associated to $\theta(t, \omega)$ by the Wick product

$$
V(t, \cdot)=\theta(t, \cdot) \diamond S(t, \cdot)=\theta_{0}(t) S_{0}(t)+\theta_{1}(t) \diamond S_{1}(t) .
$$

Similarly, if we consider a discrete time market model and keep the generalized portfolio process

$$
\theta(t)=\theta\left(t_{k}, \omega\right) ; \quad t_{k} \leq t<t_{k+1}
$$

constant from $t=t_{k}$ to $t=t_{k+1}$, the corresponding change in the generalized wealth process is

$$
\Delta V\left(t_{k}\right)=\theta\left(t_{k}\right) \diamond \Delta S\left(t_{k}\right)
$$

where

$$
\Delta V\left(t_{k}\right)=V\left(t_{k+1}\right)-V\left(t_{k}\right), \quad \Delta S\left(t_{k}\right)=S\left(t_{k+1}\right)-S\left(t_{k}\right) .
$$

If we sum this over $k$ and take the limit as $\Delta t_{k}=t_{k+1}-t_{k}$ goes to 0 , we end up with the following generalized wealth process formula

$$
V(T)=V(0)+\int_{0}^{T} \theta(t) \diamond d S(t)=V(0)+\int_{0}^{T} \theta(t) \delta S(t),
$$

where $\delta S(t)$ means that the integral is interpreted in the (Wick-Itô-)Skorohod sense.

Therefore, by (4.13)-(4.14),

$$
V(T)=V(0)+\int_{0}^{T} r \theta_{0}(t) S_{0}(t) d t+\int_{0}^{T} \mu \theta_{1}(t) \diamond S_{1}(t) d t+\int_{0}^{T} \sigma \theta_{1}(t) \diamond S_{1}(t) \delta B_{H}(t) .
$$

We now prove the fundamental result which explains why the Wick product suddenly appears in (4.21) above:

Lemma 4.4 a) Let $F, G \in(\mathcal{S})^{*}$. Then

$$
\langle F \diamond G, \psi\rangle=\langle F, \phi\rangle \cdot\langle G, \psi\rangle \quad \text { for all } \psi \in \mathcal{D} .
$$

b) Moreover, if $Z \in(\mathcal{S})^{*}$ is such that

$$
\langle Z, \psi\rangle=\langle F, \psi\rangle \cdot\langle G, \psi\rangle \quad \text { for all } \psi \in \mathcal{D}
$$

then

$$
Z=F \diamond G
$$

Proof. a) Choose $\psi=\exp ^{\diamond}\left(\int_{\mathbb{R}} h(t) d B_{H}(t)\right) \in \mathcal{D}$. We may assume that

$$
F=\exp ^{\diamond}\left(\int_{\mathbb{R}} f(t) d B_{H}(t)\right) \quad \text { and } \quad G=\exp ^{\diamond}\left(\int_{\mathbb{R}} g(t) d B_{H}(t)\right)
$$

for some $f, g \in L_{H}^{2}(\mathbb{R})$, because the set of all linear combinations of such Wick exponentials is dense in $(\mathcal{S})^{*}$. For such $F, G, \psi$ we have

$$
\langle F, \psi\rangle=E[F \cdot \psi] \quad \text { and } \quad\langle G, \psi\rangle=E[G \cdot \psi] .
$$


Therefore

$$
\begin{aligned}
& \langle F \diamond G, \psi\rangle=E\left[\exp ^{\diamond}\left(\int_{\mathbb{R}}(f+g) d B_{H}\right) \cdot \exp ^{\diamond}\left(\int_{\mathbb{R}} h d B_{H}\right)\right] \\
& =E\left[\exp \left(\int_{\mathbb{R}}(f+g) d B_{H}-\frac{1}{2}\|f+g\|_{L_{H}^{2}(\mathbb{R})}^{2}\right) \cdot \exp \left(\int_{\mathbb{R}} h d B_{H}-\frac{1}{2}\|h\|_{L_{H}^{2}(\mathbb{R})}^{2}\right)\right] \\
& =E\left[\operatorname { e x p } \left(\int_{\mathbb{R}}(f+g+h) d B_{H}\right.\right. \\
& \left.\left.\quad-\frac{1}{2}\|f\|_{L_{H}^{2}(\mathbb{R})}^{2}-\frac{1}{2}\|g\|_{L_{H}^{2}(\mathbb{R})}^{2}-\frac{1}{2}\|h\|_{L_{H}^{2}(\mathbb{R})}^{2}-(f, g)_{L_{H}^{2}(\mathbb{R})}\right)\right] \\
& =E\left[\exp \left(\int_{\mathbb{R}}(f+g+h) d B_{H}-\frac{1}{2}\|f+g+h\|_{L_{H}^{2}(\mathbb{R})}^{2}+(f, h)_{L_{H}^{2}(\mathbb{R})}+(g, h)_{L_{H}^{2}(\mathbb{R})}\right)\right] \\
& =E\left[\exp ^{\diamond}\left(\int_{\mathbb{R}}(f+g+h) d B_{H}\right) \cdot \exp (f+g, h)_{L_{H}^{2}(\mathbb{R})}\right] \\
& =\exp (f+g, h)_{L_{H}^{2}(\mathbb{R})} .
\end{aligned}
$$

On the other hand, a similar computation gives

$$
\begin{aligned}
\langle F, \psi\rangle \cdot\langle G, \psi\rangle=E\left[\exp ^{\diamond}\left(\int_{\mathbb{R}} f d B_{H}\right) \cdot \exp ^{\diamond}\left(\int_{\mathbb{R}} h d B_{H}\right)\right] \\
\cdot E\left[\exp ^{\diamond}\left(\int_{\mathbb{R}} g d B_{H}\right) \cdot \exp ^{\diamond}\left(\int_{\mathbb{R}} h d B_{H}\right)\right] \\
=\exp (f, h)_{L_{H}^{2}(\mathbb{R})} \cdot \exp (g, h)_{L_{H}^{2}(\mathbb{R})}=\exp (f+g, h)_{L_{H}^{2}(\mathbb{R})}
\end{aligned}
$$

Comparing (4.28) and (4.29) we get a).

b) This follows from the fact that the set of linear combinations of elements of $\mathcal{D}$ is dense in $(\mathcal{S})$, and $(\mathcal{S})^{*}$ is the dual of $(\mathcal{S})$.

Remark 4.5 We emphasize that this model for $f B m$ in finance does not a priori assume that the Wick product models the growth of wealth. In fact, the Wick product comes as a mathematical consequence of the basic assumption that the observed value is the result of applying a test function to a distribution process describing in a broad sense the value of a company. This way of thinking stems from microcosmos (quantum mechanics), but it has been argued that it is often a good description of macrocosmos situations as well. Here is an example:

An agent from an opinion poll firm stops a man on the street and asks him what political party he would vote for if there was an election today. Often this man on the street does not really have a firm opinion about this beforehand (he is in a diffuse state of mind politically), but the contact with the agent forces him to produce an answer. In a similar sense the general state of a company does not really have a noted stock price a priori, but brings out a number (price) when confronted with a market observer (the stock market). 
In view of the above we now make the following definitions:

Definition 4.6 a) The total wealth process $V^{\theta}(t)$ corresponding to a portfolio $\theta(t)$ in the Wick-Skorohod model is defined by

$$
V^{\theta}(t)=\theta(t) \diamond S(t)
$$

b) A portfolio $\theta(t)$ is called Wick-Skorohod self-financing if

$$
\delta V^{\theta}(t)=\theta(t) \delta S(t)
$$

i.e.

$$
V^{\theta}(t)=V^{\theta}(0)+\int_{0}^{t} \theta_{0}(s) d S_{0}(s)+\int_{0}^{t} \theta_{1}(s) \delta S_{1}(s) .
$$

In particular, we assume that the two integrals in (4.32) exist.

By the Girsanov theorem for $f B m$ (see e.g. [Mo], [V], [EvdV], [HØ]) there exists a probability measure $Q$ on $(\Omega, \mathcal{F})$ such that $Q$ is equivalent to $P$ (i.e. $Q$ has the same null sets as $P$ ) and such that

$$
\hat{B}_{H}(t):=\frac{\mu-r}{\sigma} t+B_{H}(t)
$$

is a fractional Brownian motion w.r.t. $Q$.

Replacing $B_{H}(t)$ by $\hat{B}_{H}(t)$ in $(4.32)$ we get

$$
e^{-r t} V^{\theta}(t)=V^{\theta}(0)+\int_{0}^{t} e^{-r s} \sigma \theta_{1}(s) \diamond S_{1}(s) \delta \hat{B}_{H}(s) .
$$

Definition 4.7 We call a portfolio $\theta(t)$ Wick-Skorohod admissible if it is Wick-Skorohod self-financing and $\theta_{1}(s) \diamond S_{1}(s)$ is Skorohod integrable w.r.t. $\hat{B}_{H}(s)$.

Definition 4.8 A Wick-Skorohod admissible portfolio $\theta(t)$ is called a strong arbitrage if the corresponding total wealth process $V^{\theta}(t)$ satisfies

$$
\begin{aligned}
& V^{\theta}(0)=0 \\
& V^{\theta}(T) \in L^{2}(Q) \quad \text { and } \quad V^{\theta}(T) \geq 0 \quad \text { a.s. } P \\
& P\left[V^{\theta}(T)>0\right]>0 .
\end{aligned}
$$

The following result was first proved by $[\mathrm{H} \varnothing]$ for the case $\frac{1}{2}<H<1$ and then extended to arbitrary $H \in(0,1)$ by $[\mathrm{EvdH}]$ (in a related model):

Theorem 4.9 There is no strong arbitrage in the Wick-Skorohod fractional Black-Scholes market (4.13)-(4.14). 
ProOF. If we take the expectation with respect to $Q$ of both sides of (4.34) with $t=T$ we get, by (3.14),

$$
e^{-r T} E_{Q}\left[V^{\theta}(T)\right]=V^{\theta}(0) .
$$

From this we see that (4.35)-(4.37) cannot hold.

Remark 4.10 Note that the non-existence of a strong arbitrage in this market (where the value process $S_{1}(t)$ is not a semimartingale) is not in conflict with the result of [DS] mentioned in Remark 4.2, because in this market the underlying products are Wick products, not ordinary pathwise products.

We proceed to discuss completeness in this market:

Definition 4.11 The market is called (Wick-Skorohod) complete if for every $\mathcal{F}_{T}^{(H)}$-measurable random variable $F \in L^{2}(Q)$ there exists an admissible portfolio $\theta(t)=\left(\theta_{0}(t), \theta_{1}(t)\right)$ such that

$$
F=V^{\theta}(T) \text { a.s. }
$$

By (4.34) we see that this is equivalent to requiring that there exists $\phi$ such that

$$
e^{-r T} F(\omega)=e^{-r T} E_{Q}[F]+\int_{0}^{T} \phi(s, \omega) \delta \tilde{B}_{H}(s),
$$

where

$$
\phi(s)=e^{-r s} \sigma \theta_{1}(s) \diamond S_{1}(s) .
$$

If such a $\phi$ can be found, then we put

$$
\theta_{1}(s)=\sigma^{-1} e^{r s} S_{1}(s)^{\diamond(-1)} \diamond \phi(s) .
$$

It was proved by $[\mathrm{H} \varnothing]$ (for $\frac{1}{2}<H<1$ ) and subsequently by [EvdH] in a related market (for arbitrary $H \in(0,1))$ that this market is complete. In fact, we have

Theorem $4.12([\mathbf{H} \emptyset],[\mathbf{E v d H}]) \quad$ Let $F \in L^{2}(Q)$ be $\mathcal{F}_{T}^{(H)}$-measurable. Then $F=V^{\theta}(T)$ a.s. for $\theta(t)=\left(\theta_{0}(t), \theta_{1}(t)\right)$, with

$$
\theta_{1}(t)=\sigma^{-1} e^{-\rho(T-t)} S_{1}(t)^{\diamond(-1)} \diamond \tilde{E}_{Q}\left[\hat{D}_{t}^{(H)} F \mid \mathcal{F}_{t}^{(H)}\right],
$$

where $\tilde{E}_{Q}[\cdot \mid \cdot]$ denotes the quasi-conditional expectation and $\hat{D}_{t}^{(H)}$ is the fractional HidaMalliavin derivative with respect to $\hat{B}_{H}(\cdot)$. (See [HØ] and [EvdH] for details). The other component, $\theta_{0}(t)$, is then uniquely determined by the self-financing condition (4.32).

In the Markovian case, i.e. when

$$
F(\omega)=f\left(B_{H}(T)\right)
$$

for some function $f: \mathbb{R} \rightarrow \mathbb{R}$, we can give a more explicit expression for the replicating portfolio $\theta(t)$. This is achieved by using the following representation theorem, due to $\mathrm{C}$. Bender [B1]. It has the same form as in the well-known classical case $\left(H=\frac{1}{2}\right)$ : 
Theorem $4.13([\mathrm{~B} 1])$ Let $f: \mathbb{R} \rightarrow \mathbb{R}$ be such that

$$
E\left[f^{2}\left(B_{H}(T)\right)\right]<\infty .
$$

Then

$$
f\left(B_{H}(T)\right)=E[f(B(T))]+\int_{0}^{T} \phi(t, \omega) d B_{H}(t)
$$

where

$$
\phi(t, \omega)=\left(\frac{\partial}{\partial x} E\left[f\left(x+B_{H}(T-t)\right)\right]\right)_{x=B_{H}(t)} .
$$

In view of the interpretation of the observed wealth $\hat{V}(t)$ as the result of applying a test function $\psi \in \mathcal{D}$ to the general wealth process $V(t)$, i.e.

$$
\hat{V}(t)=\langle V(t), \psi\rangle
$$

the following alternative definition of an arbitrage is natural (compare with Definition 4.8):

Definition 4.14 A Wick-Skorohod admissible portfolio $\theta(t)$ is called a weak arbitrage if the corresponding total wealth process $V^{\theta}(t)$ satisfies

$$
\begin{array}{ll}
V^{\theta}(0)=0 & \\
\left\langle V^{\theta}(T), \psi\right\rangle \geq 0 & \text { for all } \psi \in \mathcal{D} \\
\left\langle V^{\theta}(T), \psi\right\rangle>0 & \text { for some } \psi \in \mathcal{D} .
\end{array}
$$

Do weak arbitrages exist? The answer is yes. Here is an example, due to C. Bender [B3]:

Example 4.15 ([B3] A weak arbitrage) For $\varepsilon>0$ define

$$
K_{\varepsilon}(x)=\left\{\begin{array}{lll}
-1 & \text { if } & |x| \leq \varepsilon \\
1 & \text { if } & |x|>\varepsilon
\end{array}\right.
$$

Then there exists $\varepsilon_{0}>0$ such that

$$
\int_{\mathbb{R}} K_{\varepsilon_{0}}(x) \exp \left(-\frac{1}{2} x^{2}\right) d x=0 .
$$

By a variant of Lemma 2.6 in [B2] we have

$$
\begin{aligned}
& E\left[K(\langle\omega, f\rangle) \exp \left(\langle\omega, g\rangle-\frac{1}{2}\|g\|_{L_{H}^{2}(\mathbb{R})}^{2}\right)\right] \\
& =(2 \pi)^{-1 / 2}\|f\|_{L_{H}^{2}(\mathbb{R})} \int_{\mathbb{R}} K(u) \exp \left(-\frac{\left(u-(f, g)_{L_{H}^{2}(\mathbb{R})}\right)^{2}}{2\|f\|_{L_{H}^{2}(\mathbb{R})}^{2}}\right),
\end{aligned}
$$


for all bounded $K: \mathbb{R} \rightarrow \mathbb{R}, f, g \in L_{H}^{2}(\mathbb{R})$.

Applying (4.51) to $f=\mathcal{X}_{[0,1]}$ and $\langle\omega, f\rangle=B_{H}(1)$ we get

$$
\begin{aligned}
& E\left[K_{\varepsilon_{0}}\left(B_{H}(1)\right)\right]=0 \\
& E\left[K_{\varepsilon_{0}}\left(B_{H}(1)\right) \exp \left(\langle\omega, g\rangle-\frac{1}{2}\|g\|_{L_{H}^{2}(\mathbb{R})}^{2}\right] \geq 0 \quad \text { for all } g \in L_{H}^{2}(\mathbb{R})\right. \\
& E\left[K_{\varepsilon_{0}}\left(B_{H}(1)\right) \exp \left(\left\langle\omega, \mathcal{X}_{[0,1]}\right\rangle-\frac{1}{2}\left\|\mathcal{X}_{[0,1]}\right\|_{L_{H}^{2}(\mathbb{R})}^{2}\right]>0 .\right.
\end{aligned}
$$

Now consider the Skorohod fractional market (4.13)-(4.14) with $r=\mu=0, \sigma=T=1$. Then $S_{0}(t)=1$ and $S_{1}(t)=x \exp \left(B_{H}(1)-\frac{1}{2}\right)$. Moreover, $\tilde{B}_{H}(t)=B_{H}(t)$ and $P=Q$. Hence by Theorem 4.12 and (4.50) there exists a Skorohod self-financing portfolio $\theta(t)=\left(\theta_{0}(t), \theta_{1}(t)\right)$ such that

$$
K_{\varepsilon_{0}}\left(B_{H}(1)\right)=V^{\theta}(1)=\int_{0}^{T} \theta_{1}(s) \delta S(s) \quad \text { a.s. }
$$

Then $V^{\theta}(0)=0$ and by (4.53), (4.54) and (4.17) we see that (4.47) and (4.48) hold. Hence $\theta(t)$ is a weak arbitrage.

\section{A connection between the pathwise and the Wick-Skorohod model}

In spite of the fundamental differences in the features of the pathwise model and the WickSkorohod model, it turns out that there is a close relation between them. Assume $H \in\left(\frac{1}{2}, 1\right)$.

Fix $\psi \in \mathcal{D}$ and define the function $b_{H}:[0, T] \rightarrow \mathbb{R}$ by

$$
b_{H}(t)=\left\langle B_{H}(t), \psi\right\rangle=E\left[B_{H}(t) \cdot \psi\right] .
$$

Then for $p>1$ and any partition $\mathcal{P}: 0=t_{0}<t_{1}<\cdots<t_{N}=T$ of $\left.\mid 0, T\right]$ we have

$$
\begin{gathered}
\sum_{j=0}^{N-1}\left|b_{H}\left(t_{j+1}\right)-b_{H}\left(t_{j}\right)\right|^{p}=\sum_{j=0}^{N-1}\left|E\left[\left(B_{H}\left(t_{j+1}\right)-B_{H}\left(t_{j}\right)\right) \cdot \psi\right]\right|^{p} \\
\leq \sum_{j=0}^{N-1}\left(E\left[\left|B_{H}\left(t_{j+1}\right)-B_{H}\left(t_{j}\right)\right|^{p}\right]^{1 / p} \cdot E\left[\psi^{q}\right]^{1 / q}\right)^{p} \\
\leq C \sum_{j=0}^{N-1} E\left[\left|B_{H}\left(t_{j+1}\right)-B_{H}\left(t_{j}\right)\right|^{p}\right],
\end{gathered}
$$

where $\frac{1}{p}+\frac{1}{q}=1$.

Hence, by a known property of $f B m$,

$$
\sup _{\mathcal{P}} \sum_{j=0}^{N-1}\left|b_{H}\left(t_{j+1}\right)-b_{H}\left(t_{j}\right)\right|^{p}<\infty \quad \text { iff } p \geq \frac{1}{H} .
$$

In this sense the continuous function $b_{H}(t)$ is at least as regular as a generic path of a fractional Brownian motion $B_{H}(t, \omega)$. Therefore we can define integration with respect to 
$b_{H}(t)$ just as we define pathwise integration with respect to $B_{H}(t)$. Now suppose we start with the wealth generating formula in the Wick-Skorohod model

$$
V^{\theta}(T)=V^{\theta}(0)+\int_{0}^{T} \phi(s, \omega) \delta B_{H}(s) .
$$

Suppose $\phi$ is caglad and $\psi \in \mathcal{D}$. Then this gives

$$
\begin{aligned}
\hat{V}^{\theta}(T) & =\left\langle V^{\theta}(T), \psi\right\rangle=V^{\theta}(0)+\left\langle\int_{0}^{T} \phi(s, \omega) \delta B_{H}(s), \psi\right\rangle \\
& =V^{\theta}(0)+\lim _{\Delta t_{j} \rightarrow 0}\left\langle\sum_{j=0}^{N-1} \phi\left(t_{j}\right) \diamond\left(B_{H}\left(t_{j+1}\right)-B_{H}\left(t_{j}\right)\right), \psi\right\rangle \\
& =V^{\theta}(0)+\lim _{\Delta t_{j} \rightarrow 0} \sum_{j=0}^{N-1}\left\langle\phi\left(t_{j}\right), \psi\right\rangle\left\langle B_{H}\left(t_{j+1}\right)-B_{H}\left(t_{j}\right), \psi\right\rangle \\
& =V^{\theta}(0)+\lim _{\Delta t_{j} \rightarrow 0} \sum_{j=0}^{N-1} \hat{\phi}\left(t_{j}\right)\left(b_{H}\left(t_{j+1}\right)-b_{H}\left(t_{j}\right)\right) \\
& =V^{\theta}(0)+\int_{0}^{T} \hat{\phi}(t) d b_{H}(t) .
\end{aligned}
$$

We can summarize this as follows:

Theorem 4.16 If $H>\frac{1}{2}$ the mapping $F \rightarrow\langle F, \psi\rangle ; F \in L^{2}(P)$ transforms the WickSkorohod fractional Brownian motion model into the pathwise fractional Brownian motion model.

If $H=\frac{1}{2}$ this mapping transforms the Wick-Skorohod Brownian motion model into the classical Brownian motion model.

\section{Concluding remarks}

At first glance there seems to be a disagreement between the existence of arbitrage in the (fractional) pathwise model (see Theorem 4.3) and the non-existence of a (strong) arbitrage in the Wick-Skorohod model (Theorem 4.9). The above discussion, including in particular Theorem 4.16, serves to explain this apparent contradiction: The arbitrages in the pathwise model correspond to the weak arbitrages in the Wick-Skorohod model (see Example 4.15), and not to the (non-existent) strong arbitrages.

In spite of the mathematical coherence of the Wick-Skorohod model, there is still a lot of controversy about its economic interpretation and features. We refer to the discussions in $[\mathrm{BH}]$ and $[\mathrm{SV}]$ for more details.

\section{Acknowledgments}

I am grateful to Christian Bender, Tomas Björk, Nils Christian Framstad, Walter Schachermayer and John van der Hoek for helpful communication. 


\section{References}

[B1] C. Bender: An Itô formula for generalized functionals of a fractional Brownian motion with arbitrary Hurst parameter. Stoch. Proc. and their Appl. 104 (2003), 81-106.

[B2] C. Bender: The fractional Itô integral, change of measure and absence of arbitrage. Manuscript 2002.

[B3] C. Bender: Construction of a weak arbitrage. Manuscript, May 2003.

$[\mathrm{BH}]$ T. Björk and H. Hult: A note on the Wick products and the fractional Black-Scholes model. Finance \& Stochastics (to appear).

[BHØZ] F. Biagini, Y, Hu, B. Øksendal and T. Zhang: Fractional Brownian Motion and Applications. (Forthcoming book, Springer-Verlag).

[BØ] F. Biagini and B. Øksendal: Forward integrals and an Itô formula for fractional Brownian motion. Preprint, Dept. of Mathematics, University of Oslo 22/2004.

[BØSW] F. Biagini, B. Øksendal, A. Sulem and N. Wallner: An introduction to white noise theory and Malliavin calculus for fractional Brownian motion. The Proceedings of the Royal Society 460 (2004), 347-372.

[BSZ] D. Brody, J. Syroka and M. Zervos: Dynamical pricing of weather derivatives. Quantitative Finance 2 (2002), 189-198.

[CQ] L. Coutin and Z. Qian: Stochastic analysis, rough path analysis and fractional Brownian motions. Prob. Theory Related Fields 122 (2002), 108-140.

[D] A. Dasgupta: Fractional Brownian motion: Its properties and applications to stochastic integration. Ph. D. thesis, Dept. of Statistics, Univ. of North Carolina at Chapel Hill 1997.

[DHP] T. E. Duncan, Y. Hu and B. Pasik-Duncan: Stochastic calculus for fractional Brownian motion. I. Theory, Siam J. Contr. Optim. 38 (2000), 582-612.

[DS] F. Delbaen and W. Schachermayer: A general verion of the fundamental theorem of asset pricing. Mathematische Annalen 300 (1994), 463-520.

[DÜ] L. Decreusefond and A. S. Üstünel: Stochastic analysis of the fractional Brownian motion. Potential Anal. 10 (1998), 177-214.

[EvdH] R. Elliott and J. van der Hoek: A general fractional white noise theory and applications to finance. Math. Finance 13 (2003), 301-330.

[GNRV] M. Gradinaru, I. Nourdin, F. Russo and P. Vallois: $m$-order integrals and generalized Itô's formula: the case of a fractional Brownian motion with any Hurst index. Preprint, Univ. H. Poincaré Nancy 48/2002. 
[H1] Y. Hu: Option pricing in a market where the volatility is driven by fractional Brownian motions. Recent Developments in Mathematical Finance, World Scientific 2002, pp. 4959.

[H2] Y. Hu: Integral transformations and anticipative calculus for fractional Brownian motion. Manuscript 2003.

[HKPS] T. Hida, H.-H. Kuo, J. Potthoff and L. Streit: White Noise Analysis. Kluwer 1993.

[HØ] Y. Hu and B. Øksendal: Fractional white noise calculus and application to finance. Inf. Dim. Anal. Quantum Probab. Rel. Top. 6 (2003), 1-32.

[HØUZ] H. Holden, B. Øksendal, J. Ubøe and T. Zhang: Stochastic Partial Differential Equations. Birkhäuser 1996.

[K] H.-H. Kuo: White Noise Distribution Theory. CRC Press 1996.

[Mi] Y. Mishura: Fractional stochastic integration and Black-Scholes equation for fractional Brownian model with stochastic volatility. Manuscript, December 2002.

[Mo] G. Molchan: Gaussian processes with spectra which are asymptotically equivalent to a power of $\lambda$. Theory Probab. Applic. 14 (1969), 530-530.

[N] R. Norvaisa: Modelling of stock price changes. A real analysis approach. Finance \& Stochastics 4 (2000), 343-369.

[Ne] C. Necula: Option pricing in a fractional Brownian motion environment. Manuscript, February 2002.

[Nu1] D. Nualart: The Malliavin Calculus and Related Topics. Springer-Verlag 1995.

[Nu2] D. Nualart: Stochastic integration with respect to fractional Brownian motion and applications. Preprint 2004.

[NP] D. Nualart and E. Pardoux: Stochastic calculus with anticipating integrands. Probab. Th. Rel. Fields 78 (1988), 555-581.

[NVV] I. Norros, E. Valkeila and J. Virtamo: On elementary approach to a Girsanov formula and other analytical results on fractional Brownian motions. Bernoulli 5 (1999), 571578.

[R] L.C. Rogers: Arbitrage with fractional Brownian motion. Math. Finance 7 (1997), 95105.

[RV] F. Russo and P. Vallois: Stochastic calculus with respect to continuous finite quadratic variation processes. Stochastics and Stochastics Reports 70 (2000), 1-40.

[Sa] D. M. Salopek: Tolerance to arbitrage. Stoch. Proc. and their Appl. 76 (1998), 217-230.

[Sh1] A. Shiryaev: On arbitrage and replication for fractal models. In A. Shiryaev and A. Sulem (eds.): Workshop on Mathematical Finance, INRIA, Paris 1998. 
[Sh2] A. Shiryaev: Essentials of Stochastic Finance. World Scientific 1999.

[Si] I. Simonsen: Measuring anti-correlations in the Nordic electricity spot market by wavelets. Physica A 322 (2003), 597-606.

[SV] T. Sottinen and E. Valkeila: On arbitrage and replication in the fractional Black-Scholes pricing model. Statist. Decisions 21 (2003), 93-108.

[V] E. Valkeila: On some properties of geometric fractional Brownian motion. Preprint, Univ. of Helsinki, May 1999.

[vdH] J. v. d. Hoek: Private communication. 